\title{
ON INTEGRAL POINTS ON DEGREE FOUR DEL PEZZO SURFACES
}

\author{
JÖRG JAHNEL ${ }^{\ddagger}$ AND DAMARIS SCHINDLER ${ }^{*, \ddagger}$
}

\begin{abstract}
We report on our investigations concerning algebraic and transcendental Brauer-Manin obstructions to integral points on complements of a hyperplane section in degree four del Pezzo surfaces. We discuss moreover two concepts of an obstruction at an archimedean place. Concrete examples are given of pairs of non-homogeneous quadratic polynomials in four variables representing $(0,0)$ over $\mathbb{Q}$ and over $\mathbb{Z}_{p}$ for all primes $p$, but not over $\mathbb{Z}$. By blow-up, these yield cubic polynomials in three variables all integral solutions of which satisfy a gcd condition.
\end{abstract}

\section{INTRODUCTION}

One says that a variety over a number field $k$ satisfies the Hasse principle if the existence of a rational point over every completion $k_{\nu}$ of $k$ is enough to imply the existence of a $k$-rational point. This principle takes its name from the classical Hasse-Minkowski theorem, which states that the Hasse principle holds for the class of quadric hypersurfaces. Already for cubic curves and cubic surfaces however, the Hasse principle can fail. There has been much work on constructing and controlling such failures, particularly in recent time.

Many but not all (cf. [Sk99, Theorem 2]) examples of failures of the Hasse principle that are known today can be explained by one and the same argument, which was discovered by Yu. I. Manin [Ma] around 1970. In fact, a global Brauer class $\alpha \in \operatorname{Br}(X)$ is usually responsible for the non-existence of $k$-rational points on the underlying proper variety $X$ over $k$. This phenomenon is called the Brauer-Manin obstruction to rational points. Its mechanism works as follows.

Let $\mathfrak{p}$ be an arbitrary prime ideal of $k$. The Grothendieck-Brauer group is a contravariant functor from the category of schemes to the category of abelian groups. In particular, given a scheme $X$ and a $k_{\nu_{\mathrm{p}}}$-rational point $x$ : Spec $k_{\nu_{\mathrm{p}}} \rightarrow X$, there is a restriction homomorphism $x^{*}: \operatorname{Br}(X) \rightarrow \operatorname{Br}\left(k_{\nu_{\mathfrak{p}}}\right) \cong \mathbb{Q} / \mathbb{Z}$. For a Brauer class

${ }^{*}$ The second author was supported by the NSF under agreement No. DMS-1128155.

${ }^{\ddagger}$ All computations are with magma [BCP].

Date: July 28, 2017.

2010 Mathematics Subject Classification. Primary 14G05; Secondary 11D85, 11E12, 14P25, 14F22, 14G25, 14J20.

Key words and phrases. Integral point, Integral Hasse principle, Obstruction at an archimedean place, Brauer-Manin obstruction, transcendental Brauer class, degree four del Pezzo surface, $\log K 3$ surface. 
$\alpha \in \operatorname{Br}(X)$, one calls

$$
\mathrm{ev}_{\alpha, \nu_{\mathfrak{p}}}: X\left(k_{\nu_{\mathfrak{p}}}\right) \longrightarrow \mathbb{Q} / \mathbb{Z}, \quad x \mapsto x^{*}(\alpha),
$$

the local evaluation map, associated with $\alpha$. Analogously, when $\nu$ is a real place, there is the local evaluation map $\operatorname{ev}_{\alpha, \nu}: X\left(k_{\nu}\right) \rightarrow \frac{1}{2} \mathbb{Z} / \mathbb{Z}$ while, for a complex place, one has the zero map.

If $X$ is a proper $k$-scheme then the local evaluation maps are continuous with respect to the $\nu$-adic topologies on $X\left(k_{\nu}\right)$. Moreover, $\mathrm{ev}_{\alpha, \nu}$ is constant for all but finitely many places [Ja, Chapter IV, Proposition 2.3.a.ii)]. Thus, only adelic points $x=\left(x_{\nu}\right)_{\nu \in \Omega} \in X\left(\mathbb{A}_{k}\right)$ satisfying $\sum_{\nu \in \Omega} \mathrm{ev}_{\alpha, \nu}\left(x_{\nu}\right)=0 \in \mathbb{Q} / \mathbb{Z}$ may possibly be approximated by $k$-rational points.

\section{Brauer-Manin obstruction to integral points.}

The case of a non-proper variety $U$ has been studied only much more recently. Here, for $\alpha \in \operatorname{Br}(U)$, there is usually no finite set $S$ of places such that $\mathrm{ev}_{\alpha, \nu}$ would be constant for all $\nu \notin S$. Thus, the local evaluation maps seem to be useless.

The picture changes however when integral points are considered instead. So let $U$ be quasi-projective over $k$ and $\mathscr{U}$ be a model of $U$ that is defined over $\operatorname{Spec} \mathscr{O}_{k}$, for $\mathscr{O}_{k}$ the ring of integers in $k$. Then, similarly to the above, there exists a finite set $S=S_{\mathscr{U}, \alpha}[\mathrm{Sk} 01, \S 5.2]$ of places, including all archimedean ones, such that the restriction

$$
\left.\operatorname{ev}_{\alpha, \nu}\right|_{\mathscr{U}\left(\mathscr{O}_{k, \nu}\right)}: \mathscr{U}\left(\mathscr{O}_{k, \nu}\right) \longrightarrow \mathbb{Q} / \mathbb{Z}
$$

is the zero map for every place $\nu \notin S$. Consequently, for the set of all $S$-integral points, one has the inclusions (cf. [CX, §1])

$$
\mathscr{U}\left(\mathscr{O}_{k, S}\right) \subset\left(\prod_{\nu \in S} U\left(k_{\nu}\right) \times \prod_{\nu \notin S} \mathscr{U}\left(\mathscr{O}_{k, \nu}\right)\right)^{\mathrm{Br}(U)} \subset \prod_{\nu \in S} U\left(k_{\nu}\right) \times \prod_{\nu \notin S} \mathscr{U}\left(\mathscr{O}_{k, \nu}\right)
$$

for

$$
\begin{gathered}
\left(\prod_{\nu \in S} U\left(k_{\nu}\right) \times \prod_{\nu \notin S} \mathscr{U}\left(\mathscr{O}_{k, \nu}\right)\right)^{\operatorname{Br}(U)}:= \\
\left\{\left(x_{\nu}\right)_{\nu} \in \prod_{\nu \in S} U\left(k_{\nu}\right) \times \prod_{\nu \notin S} \mathscr{U}\left(\mathscr{O}_{k, \nu}\right) \mid \forall \alpha \in \operatorname{Br}(U): \sum_{\nu} \operatorname{ev}_{\alpha, \nu}\left(x_{\nu}\right)=0\right\} .
\end{gathered}
$$

In very much the same way as Yu. I. Manin did in his book [Ma] for counterexamples to the Hasse principle for rational points, J.-L. Colliot-Thélène and F. Xu [CX] explained many classical counterexamples to the integral Hasse principle or strong approximation (cf. [DW, Definition 2.1] or [PR, §7.1]) off certain primes by the Brauer-Manin obstruction to integral points. In $[\mathrm{Xu}], \mathrm{F}$. Xu even succeded in showing that the Brauer-Manin obstruction is the only obstruction to strong approximation off infinity for certain quadric fibrations, given that the set of real points is non-compact. 
Colliot-Thélène and $\mathrm{Xu}$ already considered not only algebraic, but also transcendental Brauer classes in their work (see Remark 2.11 and Remark 3.8 in [CX]), and more examples have been constructed recently by A. Kresch and Yu. Tschinkel [KT].

\section{Obstruction at infinity.}

When asking for solutions in integers, there is another point to be considered, besides local solubility and the Brauer-Manin obstruction. Consider, for example, the equation $X^{2}+23 Y^{2}=2$, which is insoluble in $\mathbb{Z}^{2}$, although there exist rational solutions, as well as $p$-adic solutions for every prime $p$. The most natural argument to show insolubility over $\mathbb{Z}$ is certainly to notice that the equation defines a compact submanifold of $\mathbb{R}^{2}$, which, as integral points are sought for, leaves only finitely many cases. The same effect occurs for every system of equations in an arbitrary number of variables that includes $Q\left(X_{1}, \ldots, X_{m}\right)=C$, for $Q$ a definite quadratic form. Cf. [CW, Exemple 5.9], which is exactly of this type. Such examples are obstructed at the real place. And they are in fact obstructed in the crudest possible sense.

On the other hand, consider the equation $X^{2}-Y^{2}=2$. Here, the submanifold defined by the equation is non-compact, but still there is an obstruction at the real place, in the sense below. The equation implies that $|X-Y| \leq \sqrt{2}$ or $|X+Y| \leq \sqrt{2}$. Thus, considering only integral points, one is reduced to finitely many algebraic subvarieties of lower dimension. The same effect occurs, of course, whenever an equation of the form $F_{1}\left(X_{1}, \ldots, X_{m}\right) F_{2}\left(X_{1}, \ldots, X_{m}\right)=C$ is considered, for $F_{1}$ and $F_{2}$ coprime, non-constant forms in an arbitrary number of variables.

When working over a number field, the latter type of obstruction may even occur at a complex place. For example, every solution in $\mathbb{Z}[i]^{2}$ of the equation $X^{2}+Y^{2}=C$ fulfils either $|X-i Y| \leq \sqrt{|C|}$ or $|X+i Y| \leq \sqrt{|C|}$.

Concerning the concept of unobstructedness at infinity, it is our impression that a definite form for it has yet to be found. We think, however, that the examples just considered, despite being so simple, are typical in a certain sense. Thus, in section 2 , we suggest two notions, called weak and strong unobstructedness, that reflect the effects just indicated, and compare them to results existing in the literature. We also show that the assumption of strong unobstructedness is in some sense necessary, when one asks for strong approximation or Zariski-density of integral points.

\section{Our results.}

The goal of this article is to present some theory and more examples, in which strong approximation off $\{\infty\}$, strong approximation off a larger set of primes, or even the integral Hasse principle are violated. We consider open subschemes of del Pezzo surfaces of degree four that are complements of an effective divisor from the anticanonical class. These are, in particular, affine log $K 3$ surfaces of Picard rank 5. Contrary to the examples previously studied, in this case both algebraic and transcendental Brauer classes may well occur. 
The article is organised as follows. Below this introduction, we first deal with obstructions at infinity (Section 2), followed by generalities about degree four del Pezzo surfaces and complements of hyperplane sections through them (Section 3), as well as the Brauer-Manin obstruction to integral points (Section 4). We compute the list of all groups that may occur as the algebraic part of the Brauer groups of the surfaces considered in Theorem 4.8. This turns out to comprise $0, \mathbb{Z} / 2 \mathbb{Z},(\mathbb{Z} / 2 \mathbb{Z})^{2}$, $(\mathbb{Z} / 2 \mathbb{Z})^{3},(\mathbb{Z} / 2 \mathbb{Z})^{4}, \mathbb{Z} / 4 \mathbb{Z}, \mathbb{Z} / 2 \mathbb{Z} \times \mathbb{Z} / 4 \mathbb{Z}$, and $(\mathbb{Z} / 2 \mathbb{Z})^{2} \times \mathbb{Z} / 4 \mathbb{Z}$, which is much richer than the corresponding list in the case of a proper degree four del Pezzo surface. For our results on the transcendental part of the Brauer group, see Theorem 4.9 and Corollary 4.10.

Section 5 then deals with the question how to evaluate a Brauer class practically. We present model cases, in which this may be done in satisfactory manner. Finally, Sections 6, 7, and 8 are devoted to explicit examples.

In a classical language, these may be formulated in the way below. A pair of (non-homogeneous) quadratic polynomials $q_{1}, q_{2} \in \mathbb{Z}\left[X_{0}, \ldots, X_{3}\right]$ in four variables does not represent $(0,0)$ in integers, although the system of equations

$$
\begin{aligned}
& q_{1}\left(X_{0}, \ldots, X_{3}\right)=0 \\
& q_{2}\left(X_{0}, \ldots, X_{3}\right)=0
\end{aligned}
$$

is soluble over $\mathbb{Q}$, as well as over $\mathbb{Z}_{p}$ for every prime number $p$.

For instance, in Section 6, we consider the following system of equations,

$$
\begin{aligned}
X_{0} X_{1}+X_{2}^{2} & =X_{3}, \\
X_{3}\left(2 X_{1}+X_{2}+X_{3}\right)+X_{0}^{2} & =X_{1} .
\end{aligned}
$$

We show that every $\mathbb{Z}\left[\frac{1}{17}\right]$-valued solution of this system fulfils the relation $\left(x_{1}, x_{3}\right)_{2}=1$, for $(., .)_{2}$ the 2-adic Hilbert symbol, although the analogous relation is not true for all $\mathbb{Z}_{2}$-valued solutions. In particular, strong approximation off $\{17, \infty\}$ is violated.

From this system, it is easy to deduce another one that has no integral solution at all, thereby violating the integral Hasse principle. Moreover, blowing up the point $(0: 1: 0: 0: 0)$, we are led to the affine cubic surface

$$
\begin{aligned}
128 Y_{0}^{3}+144 Y_{0}^{2}+32 Y_{0} Y_{1} Y_{2}+ & 8 Y_{0} Y_{1}+128 Y_{0} Y_{2}^{2}+80 Y_{0} Y_{2}+66 Y_{0} \\
& -16 Y_{1}^{2} Y_{2}-3 Y_{1}^{2}-4 Y_{1} Y_{2}+80 Y_{2}^{2}+40 Y_{2}+12=0
\end{aligned}
$$

with the amusing property that each of its integral points must satisfy the condition that $\operatorname{gcd}\left(8 y_{0}+3,16 y_{2}+3\right)>1$.

Neither of these examples can be explained by an obstruction at the real place, even in a strong sense. Observe, for instance, that the homogeneous parts $\underline{q}_{1}, \underline{q}_{2}$ of degree 2 always generate a pencil consisting entirely of indefinite quadratic forms. This implies that the real manifold defined by $\left(q_{1}, q_{2}\right)$ is non-compact [Fi, Satz 1] (see also [TWMW, Tables 1-3]) and, hence, has limit points at the boundary. In addition to that, both our surfaces in Sections 6 and 7 are strongly unobstructed at $\infty$. 
The arithmetic behaviour we describe is entirely explained by algebraic and transcendental Brauer classes. In this respect, our examples are similar to the ones given in $[\mathrm{CX}]$, but different from those recently presented by Y. Harpaz [Harp, §4.2] and U. Derenthal and D. Wei [DW, Example 6.2].

The surface we examine in Section 8 is somewhat different. Its arithmetic is explained by Brauer classes together with effects caused by the underlying real manifold $U(\mathbb{R})$ being disconnected into non-compact components and a compact one. So, similarly to [DW, Example 6.2], an interaction of effects caused by Brauer classes occurs with an obstruction at infinity. This example shows that any reasonable definition of unobstructedness at infinity must include requirements on all connected components. In particular, it is insufficient just to require the existence of a single well-behaved one.

Acknowledgements: We thank Prof. J.-L. Colliot-Thélène for comments on an earlier version of this paper and Dr. M. Bright for pointing out an oversight in our original formulation of Corollary 4.10. The second author is supported by a NWO grant 016.Veni.173.016.

\section{Obstruction at infinity}

Example 2.1. Consider the subscheme $\mathscr{X} \subset \mathbf{P}_{\mathbb{Z}}^{4}$ defined by the system of equations

$$
\begin{aligned}
& 2 X_{0}^{2}+X_{1}^{2}+X_{2}^{2}=26 X_{4}^{2}, \\
& 3 X_{1}^{2}+X_{2}^{2}+X_{3}^{2}=13 X_{4}^{2}
\end{aligned}
$$

and put $\mathscr{U}:=\mathscr{X} \backslash \mathscr{H}$, for $\mathscr{H}:=V\left(X_{4}\right) \subset \mathbf{P}_{\mathbb{Z}}^{4}$. Then the generic fibre $X$ of $\mathscr{X}$ is a degree four del Pezzo surface. Moreover, there exist $\mathbb{Q}$-rational points on the generic fibre $U$ of $\mathscr{U}$, as well as $\mathbb{Z}_{p}$-valued points on $\mathscr{U}$ for every prime number $p$. Examples are $\left(\frac{18}{7}: \frac{1}{7}: \frac{25}{7}: \frac{3}{7}: 1\right)$ and $\left(\frac{54}{19}: \frac{23}{19}: \frac{55}{19}: \frac{9}{19}: 1\right)$. Yet, there is no integral point.

This is, however, not at all an interesting example. The reason is simply that there is an obstruction at infinity. Indeed, the two equations imply $\left|x_{0}\right|, \ldots,\left|x_{3}\right| \leq 3$ for every $\mathbb{Q}$-rational point $\left(x_{0}: \ldots: x_{3}: 1\right)$ on $\mathscr{U}$. For integral points, this leaves us with only finitely many cases and it turns out that actually none of them corresponds to an integral point. This motivates the first part of the following definition. The second part is motivated by examples of the type $X^{2}-Y^{2}=2$, as discussed in the introduction.

Definition 2.2. Let $k$ be a number field and $\nu$ an archimedean place of $k$. Furthermore, suppose that $X \subset \mathbf{P}_{k}^{n}$ is an irreducible, closed subscheme, $l \in \Gamma\left(\mathbf{P}_{k}^{n}, \mathscr{O}(1)\right)$ a linear form, and $H:=V(l) \subset \mathbf{P}_{k}^{n}$ the corresponding $k$-rational hyperplane. Put $U:=X \backslash H$.

i) We say that $U$ is weakly unobstructed at $\nu$ if every connected component of $U\left(k_{\nu}\right)$ has a limit point on $H$.

ii) We call $U$ strongly unobstructed at $\nu$ if every connected component $U^{\prime}$ of $U\left(k_{\nu}\right)$ fulfils the following condition. 
Given an integer $d>0$, finitely many $k$-rational forms $s_{1}, \ldots, s_{r} \in \Gamma(X, \mathscr{O}(d))$, neither being a scalar multiple of $l^{d}$, and a constant $c \in \mathbb{R}$, there always exists a point $x \in U^{\prime}$ such that $\left\|s_{i}(x) / l^{d}(x)\right\|_{\nu} \geq c$ for all $i \in\{1, \ldots, r\}$.

iii) If $U$ is not weakly unobstructed (strongly unobstructed) at $\nu$ then we say that $U$ is strongly obstructed (weakly obstructed) at $\nu$.

Remarks 2.3. i) As $\mathbf{P}^{n}\left(k_{\nu}\right)$ is a compact topological space, the property of being weakly unobstructed simply means that $U\left(k_{\nu}\right)$ does not have a bounded connected component. We note that if $\nu$ is a complex place and $\operatorname{dim} U \geq 1$ then $U$ is weakly unobstructed at $\nu$.

ii) On the other hand, strong unobstructedness states that no connected component of $U\left(k_{\nu}\right)$ is contained in a finite union of tubular neighbourhoods of hypersurfaces of the form $\left\|s_{i}(x) / l^{d}(x)\right\|_{\nu} \leq c$.

iii) Example 2.1 is strongly obstructed. In fact, the topological boundary of $\mathscr{U}(\mathbb{R})$ is given by $2 X_{0}^{2}+X_{1}^{2}+X_{2}^{2}=3 X_{1}^{2}+X_{2}^{2}+X_{3}^{2}=0$, which defines the empty set in $\mathbf{P}^{3}(\mathbb{R})$.

iv) As already remarked in the introduction, it seems to us that a definite form for the concept of unobstructedness at infinity has yet to be found. For instance, in [DW, Theorems 1.1 and 1.2], U. Derenthal and D. Wei included some unboundedness conditions at infinity and showed that they are, in general, necessary. In the case of a fibration over the affine line $\mathbf{A}^{1}$, our notion of being strongly unobstructed implies unboundedness in the sense of Derenthal and Wei. Our definition is partly motivated by their examples, which however come with a natural projection to some affine space. Our definition aims to suggest a general notion of unobstructedness at infinity, which does not require such a fibration.

It is inspired, too, by the results of J.-L. Colliot-Thélène and F. Xu [CX, Theorems 3.7, 4.5, and 8.3], as well as by the recent work of Y. Harpaz [Harp]. In fact, in the case that $U\left(k_{\nu}\right)$ is connected, Harpaz' concept of being split [Harp, Def. 1.0.8] is slightly stronger than weak unobstructedness. It seems, however, that split varieties do not behave well, in general, when $U\left(k_{\nu}\right)$ is disconnected, cf. Section 8 . Y. Harpaz' example of a surface that is split, has no Brauer-Manin obstruction, yet no integral point, is weakly obstructed at infinity, according to our definition, as we show below. It is exactly this property of being obstructed that is used in his work to show that the surface has no integral points.

Example 2.4 (cf. [Harp, §4.2]). Let $X \subset \mathbf{P}_{\mathbb{Q}}^{3}$ be defined by the equation

$$
\left(\left(11 X_{0}+5 X_{3}\right) X_{1}+3 X_{3}^{2}\right) X_{2}=\left(3 X_{0}+X_{3}\right) X_{3}^{2}
$$

and put $U:=X \backslash H$, for $H:=V\left(X_{3}\right) \subset \mathbf{P}_{\mathbb{Q}}^{4}$. Then $U$ is weakly unobstructed at $\infty$, but not strongly unobstructed.

Proof. It is not hard to see that $U(\mathbb{R})$ is connected. Hence, weak unobstructedness follows from $U$ being split in the terminology of [Harp, Def. 1.0.8]. 
In order to disprove strong unobstructedness, observe that every real point $x=\left(x_{0}: x_{1}: x_{2}: 1\right) \in U(\mathbb{R})$ must fulfil the equation $\left(\left(11 x_{0}+5\right) x_{1}+3\right) x_{2}=3 x_{0}+1$, i.e. $x_{0}\left(11-\frac{3}{x_{1} x_{2}}\right)=\frac{1}{x_{1} x_{2}}-\frac{3}{x_{1}}-5$. This immediately shows that $\left|x_{1}\right|,\left|x_{2}\right| \geq 1$ implies $\left|x_{0}\right| \leq \frac{9}{8}$.

Example 2.5. Let $X \subset \mathbf{P}_{\mathbb{Q}}^{6}$ be defined by the equation

$$
\left(X_{0}^{2}+X_{1}^{2}-X_{2}^{2}\right)\left(X_{3}^{2}+X_{4}^{2}-X_{5}^{2}\right)=\left(X_{0}^{2}+X_{1}^{2}-X_{2}^{2}+X_{3}^{2}+X_{4}^{2}-X_{5}^{2}\right) X_{6}^{2}
$$

and put $U:=X \backslash H$, for $H:=V\left(X_{6}\right) \subset \mathbf{P}_{\mathbb{Q}}^{4}$. Then $U$ is weakly unobstructed at $\infty$, but not strongly unobstructed.

Proof. It is not hard to see that $U(\mathbb{R})$ has exactly two connected components and that $(1: 0: 0: 0: 0: 0: 0)$ is a limit point for either of them.

On the other hand, every real point $x=\left(x_{0}: x_{1}: x_{2}: x_{3}: x_{4}: x_{5}: 1\right) \in U(\mathbb{R})$ must fulfil the equation $Q\left(x_{0}, x_{1}, x_{2}\right) Q\left(x_{3}, x_{4}, x_{5}\right)=Q\left(x_{0}, x_{1}, x_{2}\right)+Q\left(x_{3}, x_{4}, x_{5}\right)$, and hence $\left|Q\left(x_{0}, x_{1}, x_{2}\right)\right| \leq 2$ or $\left|Q\left(x_{3}, x_{4}, x_{5}\right)\right| \leq 2$ for $Q$ the ternary quadratic form $Q\left(X_{0}, X_{1}, X_{2}\right):=X_{0}^{2}+X_{1}^{2}-X_{2}^{2}$.

The next theorem shows that the notion of being strongly unobstructed is essentially necessary. In fact otherwise, under the assumption that $U(\mathbb{R})$ is connected, the integral points on $\mathscr{U}$ are contained in a union of finitely many lower-dimensional subschemes. In particular, they are not Zariski dense and strong approximation off $\{\infty\}$ cannot be fulfilled.

Theorem 2.6. Let $\mathscr{X} \subset \mathbf{P}_{\mathbb{Z}}^{n}$ be an irreducible, closed subscheme, $l \in \Gamma\left(\mathbf{P}_{\mathbb{Z}}^{n}, \mathscr{O}(1)\right)$ a linear form, $\mathscr{H}:=V(l) \subset \mathbf{P}_{\mathbb{Z}}^{n}$, and $\mathscr{U}:=\mathscr{X} \backslash \mathscr{H}$. Denote the generic fibres of $\mathscr{X}$ and $\mathscr{U}$ by $X$ and $U$, respectively.

Assume that $U(\mathbb{R})$ is connected and that $U$ is weakly obstructed at infinity. Then the set $\mathscr{U}(\mathbb{Z})$ of integral points is not Zariski-dense in $\mathscr{U}$.

Proof. Since $U$ is weakly obstructed at infinity, there exist an integer $d>0$ and forms $s_{1}, \ldots, s_{r} \in \Gamma\left(X, \mathscr{O}_{X}(d)\right)$, none of them a multiple of $l^{d}$, as well as a positive constant $c>0$, such that the following is true. For every $x \in U(\mathbb{Q})$, there is an $1 \leq i \leq r$ such that

$$
\left|\frac{s_{i}(x)}{l^{d}(x)}\right| \leq c .
$$

Taking global sections commutes with arbitrary flat base extensions. In particular, $\Gamma\left(X, \mathscr{O}_{X}(d)\right)=\Gamma\left(\mathscr{X}, \mathscr{O}_{\mathscr{X}}(d)\right) \otimes_{\mathbb{Z}} \mathbb{Q}$. As a consequence of this, we see that there exists a non-zero integer $M \in \mathbb{Z}$ such that $M s_{1}, \ldots, M s_{r} \in \Gamma\left(\mathscr{X}, \mathscr{O}_{\mathscr{X}}(d)\right)$.

Consider now an integral point $x \in \mathscr{U}(\mathbb{Z})$. Its restriction to the generic fibre has the property that

$$
\left|\frac{M s_{i}(x)}{l^{d}(x)}\right| \leq M c,
$$


for some $1 \leq i \leq r$. Thus, for every such integral point, there exists an integer $B$ such that $|B| \leq M c$ and

$$
\frac{M s_{i}(x)}{l^{d}(x)}=B
$$

In particular, this system defines a Zariski-closed subset $Z \subset U$. Moreover, if we had $Z=U$ then $s_{i}$ would be equal to $B$ on $U$, a contradiction to none of the $s_{i}$ being a scalar multiple of the form $l^{d}$. Hence, $Z \varsubsetneqq U$.

We conclude that there is a finite union of such proper Zariski-closed subsets of $U$ that contains all restrictions of integral points $P \in \mathscr{U}(\mathbb{Z})$ to the generic fibre. Hence, the integral points on $\mathscr{U}$ cannot be Zariski-dense.

We note that Theorem 2.6 may be seen as a generalisation of Theorem 4.1.1. of Harpaz's work [Harp]. Finally, we close this section in giving a useful criterion to detect varieties that are strongly unobstructed.

Theorem 2.7. Let $k$ be a number field, $\nu$ an archimedean place of $k, X \subset \mathbf{P}_{k}^{n}$ be a normal, projective variety, $l \in \Gamma\left(\mathbf{P}_{k}^{n}, \mathscr{O}(1)\right)$ a linear form, $H:=V(l) \subset \mathbf{P}_{k}^{n}$ the corresponding $k$-rational hyperplane, and put $U:=X \backslash H$.

\section{Suppose that}

- the scheme $(H \cap X)_{k_{\nu}}$ is reduced and irreducible and that

- every connected component of $U\left(k_{\nu}\right)$ has a limit point $x \in(H \cap X)\left(k_{\nu}\right)$ that is non-singular as a point on $H \cap X$.

Then $U$ is strongly unobstructed at $\nu$.

Note that Theorem 2.7 does not apply to example 2.4, despite $X$ being a normal cubic surface, since $H \cap X$ is a union of three lines.

Proof of Theorem 2.7. If $\nu$ is a complex place then $U\left(k_{\nu}\right)$ is clearly connected, but the same is not necessarily true for a real place. Thus, let $U^{\prime}$ be a connected component of $U\left(k_{\nu}\right)$. There is a limit point $x^{\prime} \in(H \cap X)\left(k_{\nu}\right)$ that is nonsingular on the limit set. Therefore, there exists a neighbourhood $V \ni x^{\prime}$ such that $\left[U^{\prime} \cup(H \cap X)\left(k_{\nu}\right)\right] \cap V$ is diffeomorphic to some $\mathbb{D}^{m}, \mathbb{R}^{m}$ or half space $\mathbb{R}^{m-1} \times \mathbb{R}_{\geq 0}$.

Now let degree $d$ forms $s_{1}, \ldots, s_{r}$ not being multiples of $l^{d}$ and a constant $c$ be given. We choose a linear form, which we denote by $l^{\prime}$, in such a way that $l^{\prime}\left(x^{\prime}\right) \neq 0$. Shrinking $V$, if necessary, we may assume that the same holds on the whole of $V$.

By our assumptions, $(H \cap X)_{k_{\nu}}$ defines a prime Weil divisor on $X_{k_{\nu}}$. Put $d_{i}:=\nu_{(H \cap X)_{k_{\nu}}}\left(s_{i}\right)$ for $i=1, \ldots, r$. Since $s_{1}, \ldots, s_{r}$ are not multiples of $l^{d}$, we have $0 \leq d_{i}<d$ for each $i$. The rational functions

$$
f_{i}:=\frac{s_{i}}{l^{d_{i}} l^{\prime d-d_{i}}}
$$

have neither zeroes nor poles along the divisor $(H \cap X)_{k_{\nu}}$.

In particular, there exists a point $x^{\prime \prime} \in(H \cap X)\left(k_{\nu}\right) \cap V$ such that $f_{i}\left(x^{\prime \prime}\right) \neq 0$ for $i=1, \ldots, r$. Say, we have $\left|s_{i}\left(x^{\prime \prime}\right) / l^{d_{i}}\left(x^{\prime \prime}\right) l^{\prime d-d_{i}}\left(x^{\prime \prime}\right)\right| \geq \varepsilon$ for every $i=1, \ldots, r$ 
and some $\varepsilon>0$, which we may assume to fulfil $\varepsilon<2 c$. Moreover, $l\left(x^{\prime \prime}\right)=0$, since $x^{\prime \prime} \in(H \cap X)$.

Next, we approximate $x^{\prime \prime}$ by a point $x^{\prime \prime \prime} \in U^{\prime} \cap V$ outside the limit set. We may enforce that $\left|s_{i}\left(x^{\prime \prime \prime}\right) / l^{d_{i}}\left(x^{\prime \prime \prime}\right) l^{\prime d-d_{i}}\left(x^{\prime \prime \prime}\right)\right| \geq \varepsilon / 2$ for every $i=1, \ldots, r$ and that $0<\left|l\left(x^{\prime \prime \prime}\right) / l^{\prime}\left(x^{\prime \prime \prime}\right)\right| \leq \frac{\varepsilon}{2 c}$. This yields

$$
\left|\frac{s_{i}\left(x^{\prime \prime \prime}\right)}{l^{d}\left(x^{\prime \prime \prime}\right)}\right|=\left|\frac{s_{i}\left(x^{\prime \prime \prime}\right)}{l_{i}\left(x^{\prime \prime \prime}\right) l^{\prime d-d_{i}\left(x^{\prime \prime \prime}\right)}}\right| \cdot\left|\frac{l^{\prime}\left(x^{\prime \prime \prime}\right)}{l\left(x^{\prime \prime \prime}\right)}\right|^{d-d_{i}} \geq \frac{\varepsilon}{2} \cdot\left(\frac{2 c}{\varepsilon}\right)^{d-d_{i}} \geq \frac{\varepsilon}{2} \cdot \frac{2 c}{\varepsilon}=c
$$

for $i=1, \ldots, r$, as required.

\section{Del Pezzo surfaces of Degree Four AND COMPLEMENTS OF HYPERPLANE SECTIONS}

We are interested in quasi-projective schemes $U \subset \mathbf{P}_{k}^{4}$, given by systems of equations of the type

$$
\begin{aligned}
Q_{1}\left(X_{0}, \ldots, X_{4}\right) & =0, \\
Q_{2}\left(X_{0}, \ldots, X_{4}\right) & =0, \\
X_{4} & \neq 0,
\end{aligned}
$$

for homogeneous quadrics $Q_{1}, Q_{2} \in \mathscr{O}_{k}\left[X_{0}, \ldots, X_{4}\right]$. Then a rational point on $U$ is the same as a solution $\left(x_{0}, \ldots, x_{3}, 1\right)$ for $x_{0}, \ldots, x_{3} \in k$, while an integral point may be thought of as a solution $\left(x_{0}, \ldots, x_{3}, 1\right)$ for $x_{0}, \ldots, x_{3} \in \mathscr{O}_{k}$. The scheme $U$ itself is an open subscheme of a del Pezzo surface of degree four.

By definition, a del Pezzo surface is a non-singular, proper algebraic surface $X$ over a field $k$ with an ample anti-canonical sheaf $\mathscr{K}^{-1}$. Over an algebraically closed field, every del Pezzo surface of degree $d \leq 7$ is isomorphic to $\mathbf{P}^{2}$, blown up in $(9-d)$ points in general position [Ma, Theorem 24.4.iii)].

According to the adjunction formula, a non-singular complete intersection of two quadrics in $\mathbf{P}^{4}$ is del Pezzo. The converse is true, as well. For every del Pezzo surface of degree four, its anticanonical image is the complete intersection of two quadrics in $\mathbf{P}^{4}$ [Do, Theorem 8.6.2].

Thus, associated with a degree four del Pezzo surface $X$, there is a pencil $\left(\mu Q_{1}+\nu Q_{2}\right)_{(\mu: \nu) \in \mathbf{P}^{1}}$ of quadrics in $\mathbf{P}^{4}$, the base locus of which is $X$. This pencil is uniquely determined up to an isomorphism of $\mathbf{P}^{4}$. It contains exactly five degenerate fibres, each of which is of rank precisely 4 [Wi, Proposition 3.26.iv)].

Over an algebraically closed field, a degree four del Pezzo surface contains exactly 16 lines [Ma, Theorem 26.2.iii)] and exactly ten one-dimensional linear systems of conics [VAV, §2.3]. The latter may be obtained as follows. Take the five degenerate quadrics in the pencil. As they are of rank 4, each of them contains two onedimensional linear systems of planes. Intersecting with a second quadric from the pencil, one finds one-dimensional linear systems of conics.

Facts 3.1 (The geometric Picard group). Let $k$ be an algebraically closed field and $X$ a del Pezzo surface of degree four over $k$. Then 
i) $\operatorname{Pic}(X) \cong \mathbb{Z}^{6}$. The classes of the 16 lines form a generating system for $\operatorname{Pic}(X)$. Moreover, the canonical class $\mathscr{K} \in \operatorname{Pic}(X)$ is not divisible by any integer $\neq \pm 1$.

ii) The orthogonal complement $\operatorname{Pic}^{0}(X):=\langle\mathscr{K}\rangle^{\perp} \subset \operatorname{Pic}(X)$ is, up to sign, isometric to the lattice $D_{5}$.

iii) The group $W$ of all automorphisms of $\mathrm{Pic}(X)$ respecting $\mathscr{K}$ and the intersection pairing is isomorphic to the Weyl group $W\left(D_{5}\right)$.

iv) The operation of $W$ permutes the classes of the 16 lines in $\operatorname{Pic}(X)$. This operation is faithful. The image of the corresponding group homomorphism $W \rightarrow S_{16}$ is the largest subgroup of $S_{16}$ that respects the intersection matrix.

$\mathrm{v})$ The operation of $W$ permutes the classes in $\operatorname{Pic}(X)$ of the 10 linear systems of conics. This operation is faithful, too. The image of the corresponding group homomorphism $W \rightarrow S_{10}$ is isomorphic to $(\mathbb{Z} / 2 \mathbb{Z})^{4} \rtimes S_{5}$. Here, $(\mathbb{Z} / 2 \mathbb{Z})^{4} \subset(\mathbb{Z} / 2 \mathbb{Z})^{5}$ means the subgroup

$$
\left\{\left(x_{0}, \ldots, x_{4}\right) \in(\mathbb{Z} / 2 \mathbb{Z})^{5} \mid x_{0}+\ldots+x_{4}=0\right\}
$$

being acted upon by $S_{5}$ in the natural way.

Proof. i) follows immediately from the description of Pic $(X)$ given in [VAV, Proposition 2.2], together with [Ma, Theorem 26.2.i)], while ii), iii) and iv) are parts of [Ma, Theorem 23.9].

Finally, v) is known for a long time, as well. It follows, for example, from the discussion in [KST, pp. 8-10].

Remark 3.2. The existence of an isomorphism $W\left(D_{5}\right) \cong(\mathbb{Z} / 2 \mathbb{Z})^{4} \rtimes S_{5}$ may, of course, be seen directly from the construction of the root system $[\mathrm{Hu}, \S 12]$. Moreover, $\operatorname{Aut}\left(D_{5}\right) \cong(\mathbb{Z} / 2 \mathbb{Z})^{5} \rtimes S_{5}$, in which $W\left(D_{5}\right)$ is a subgroup of index 2 .

Lemma 3.3. Let $k$ be an algebraically closed field, $X$ a del Pezzo surface of degree four over $k$, and $D \subset X$ an irreducible divisor such that $\mathscr{O}(-D)=\mathscr{K} \in \operatorname{Pic}(X)$ is the canonical class. Put $U:=X \backslash D$. Then

i) $\operatorname{Pic}(U) \cong \operatorname{Pic}(X) /\langle\mathscr{K}\rangle$.

ii) The orthogonal projection $\pi: \operatorname{Pic}(X) \rightarrow\langle\mathscr{K}\rangle^{\perp} \otimes_{\mathbb{Z}} \mathbb{R}$ induces an isomorphism

$$
\operatorname{Pic}(U) \stackrel{\cong}{\longrightarrow} D_{5}^{*}
$$

for $D_{5}^{*}$ the lattice dual to $D_{5}$.

Proof. i) This is [Hart, Proposition II.6.5.c)].

ii) By Fact 3.1, the kernel of $\pi$ is exactly $\langle\mathscr{K}\rangle$. Moreover, for every $\mathscr{L} \in \operatorname{Pic}(X)$, one has $\langle\pi(\mathscr{L}), \mathscr{C}\rangle=\langle\mathscr{L}, \mathscr{C}\rangle \in \mathbb{Z}$ for all $\mathscr{C} \in\langle\mathscr{K}\rangle^{\perp}=D_{5}$. Hence $\pi(\mathscr{L}) \in D_{5}^{*}$ and, consequently, $\operatorname{im} \pi \subseteq D_{5}^{*}$.

In order to see that in fact equality is true, we first recall that $D_{5}$ is of discriminant $4\left[\mathrm{Hu}, \S 11\right.$, Exc. 2]. Thus, $\langle\mathscr{K}\rangle^{\perp}$ is of discriminant $(-4)$. As $\langle\mathscr{K}, \mathscr{K}\rangle=4$, this yields that $\langle\mathscr{K}\rangle \oplus\langle\mathscr{K}\rangle^{\perp}$ is of discriminant $(-16)$. Moreover, $\operatorname{Pic}(X)$ is unimodular. 
Therefore, $\langle\mathscr{K}\rangle \oplus\langle\mathscr{K}\rangle^{\perp}$ must be of index 4 in $\operatorname{Pic}(X)$. Hence \# im $\pi / D_{5}=4$. But $\# D_{5}^{*} / D_{5}=4$, again by $[\mathrm{Hu}, \S 11$, Exc. 2].

Corollary 3.4. Let $X$ and $U$ be as above and $G$ be a group operating on the 16 lines of $X$ such that the intersection matrix is respected.

i) Then the induced operation of $G$ on $\operatorname{Pic}(X)$ respects the intersection pairing as well as the canonical class.

ii) The induced operation of $G$ on $\operatorname{Pic}(U) \cong D_{5}^{*}$ takes place via isometries. The associated homomorphism $G \rightarrow \operatorname{Aut}\left(D_{5}^{*}\right)$ factors via $W\left(D_{5}\right)$.

Proof. i) The operation of $G$ on the free $\mathbb{Z}$-module $\operatorname{Div}(X)$ over the 16 lines respects the intersection pairing and, by Fact 3.1.i), $\operatorname{Pic}(X)$ is the quotient of $\operatorname{Div}(X)$ modulo its radical. Finally, the canonical class is automatically respected, as the divisor being the sum over all 16 lines defines the invertible sheaf $\mathscr{K}^{\otimes(-4)}$.

ii) The first assertion is a direct consequence of i). The second follows from Fact 3.1.iii).

Under some minor assumptions, $U$ is an example of a $\log K 3$ surface. Let us shortly recall the definition.

Definition 3.5 (cf. [Harp, Def. 2.0.15]). A $\log K 3$ surface is a scheme $U$ that may be written in the form $U=X \backslash D$, for $X$ a non-singular, connected scheme that is proper of dimension two over a field $k$ and $D \subset X$ an effective divisor such that

i) $\mathscr{O}(-D) \in \operatorname{Pic}(X)$ is the canonical class and

ii) $\pi_{1}^{\text {ét }}\left(U_{\bar{k}},.\right)=0$.

Theorem 3.6. Let $X \subset \mathbf{P}^{4}$ a non-singular complete intersection of two quadrics over a field $k$ of characteristic 0 and $U:=X \backslash H$ be the complement of a non-singular hyperplane section. Then $U$ is a $\log K 3$ surface.

Proof. We have $\pi_{1}^{\text {ét }}\left(X_{\bar{k}}\right.$, . $)=0$, since $X_{\bar{k}}$ is isomorphic to $\mathbf{P}^{2}$, blown up in five points. Moreover, [Harp, Lemma 3.3.6] yields that $\pi_{1}^{\text {ét }}\left(U_{\bar{k}}\right.$, . $)=0$, too. Indeed, each of the 16 lines on $X_{\bar{k}}$ intersects the irreducible divisor $H \cap X$ transversely and in exactly one point. Finally, the adjunction formula shows that $\left.\mathscr{K}_{X} \cong \mathscr{O}_{\mathbf{P}^{4}}(-5 H+2 H+2 H)\right|_{X} \cong \mathscr{O}_{X}(-H)$, which implies the claim.

\section{The Grothendieck-Brauer Group}

Generalities.

By definition [GrBrII, Remarque 2.7], the cohomological Grothendieck-Brauer group $\operatorname{Br}(U)$ of a scheme $U$ is the étale cohomology group $H_{\text {ét }}^{2}\left(U, \mathbb{G}_{m}\right)$. If $U$ is defined over a field $k$ then the Hochschild-Serre spectral sequence [SGA4, Exp. VIII, Proposition 8.4]

$$
H^{p}\left(\operatorname{Gal}(\bar{k} / k), H_{\text {ét }}^{q}\left(U_{\bar{k}}, \mathbb{G}_{m}\right)\right) \Longrightarrow H_{\text {ét }}^{p+q}\left(U, \mathbb{G}_{m}\right)
$$

carries essential information about $\operatorname{Br}(U)$. It yields a three-step filtration

$$
0 \subseteq \operatorname{Br}_{0}(U) \subseteq \operatorname{Br}_{1}(U) \subseteq \operatorname{Br}(U)
$$


the subquotients of which we are going to describe below. In order to avoid unnecessary complications, let us assume that

$$
\Gamma_{\text {ét }}\left(U_{\bar{k}}, \mathbb{G}_{m}\right)=\bar{k}^{*} .
$$

Lemma 4.1. Let $k$ be a field, $X$ a non-singular, proper scheme over $k$, and $U=X \backslash D$ be the complement of a divisor D. Assume that $D$ splits geometrically into irreducible components $D_{1}, \ldots, D_{r}$, the intersection matrix of which has rank $r$.

Then (1) is fulfilled.

Proof. The group $\Gamma_{\text {ét }}\left(U_{\bar{k}}, \mathbb{G}_{m}\right)$ consists of all rational functions $s$ on $X_{\bar{k}}$ such that $\operatorname{div} s=k_{1}\left[D_{1}\right]+\ldots+k_{r}\left[D_{r}\right]$ for some integers $k_{1}, \ldots, k_{r}$. Since div $s$ is a principal divisor on $X_{\bar{k}}$, its intersection numbers with $D_{1}, \ldots, D_{r}$ must all vanish. By our assumption, this is possible only for $k_{1}=\ldots=k_{r}=0$. I.e., $s$ must be constant and one sees that $\Gamma_{\text {ét }}\left(U_{\bar{k}}, \mathbb{G}_{m}\right)=\bar{k}^{*}$, as required.

Remark 4.2. The assumptions on $D$ are satisfied, in particular, if $D$ is a geometrically irreducible divisor such that $D^{2} \neq 0$.

4.3. i) In general, $\operatorname{Br}_{0}(U)$ is the image of a natural homomorphism

$$
H^{2}\left(\operatorname{Gal}(\bar{k} / k), \Gamma_{\text {ét }}\left(U_{\bar{k}}, \mathbb{G}_{m}\right)\right) \longrightarrow \operatorname{Br}(U) .
$$

Under the assumption of (1), this shows that $\operatorname{Br}_{0}(U)$ is the image of the natural homomorphism $\operatorname{Br}(k) \rightarrow \operatorname{Br}(U)$. The homomorphism (2) is an injection, thereby ensuring that $\operatorname{Br}_{0}(U) \cong \operatorname{Br}(k)$, under some minor assumption. A sufficient condition is that $U$ has a $k$-rational point or, if $k$ is a number field, that $U$ has an adelic point (cf. [Co, Proposition 1.3.4.1]).

The part $\operatorname{Br}_{0}(U) \subseteq \operatorname{Br}(U)$ does not contribute to the Brauer-Manin obstruction.

ii) The subquotient $\operatorname{Br}_{1}(U) / \operatorname{Br}_{0}(U)$ is, in general, isomorphic to

$$
\operatorname{ker} d_{2}^{1,1}: H^{1}\left(\operatorname{Gal}(\bar{k} / k), \operatorname{Pic}\left(U_{\bar{k}}\right)\right) \longrightarrow H^{3}\left(\operatorname{Gal}(\bar{k} / k), \Gamma_{\text {ét }}\left(U_{\bar{k}}, \mathbb{G}_{m}\right)\right) \text {, }
$$

for $d_{2}^{1,1}$ the differential in the Hochschild-Serre spectral sequence.

Under the assumption of (1), the right hand side simplifies to $H^{3}\left(\operatorname{Gal}(\bar{k} / k), \bar{k}^{*}\right)$. Moreover, if $k$ is a number field then, as a by-product of class field theory [Ta, section 11.4], it is known that $H^{3}\left(\operatorname{Gal}(\bar{k} / k), \bar{k}^{*}\right)=0$. Consequently, one has in fact

$$
\operatorname{Br}_{1}(U) / \operatorname{Br}_{0}(U) \cong H^{1}\left(\operatorname{Gal}(\bar{k} / k), \operatorname{Pic}\left(U_{\bar{k}}\right)\right) \text {. }
$$

The subquotient $\operatorname{Br}_{1}(U) / \mathrm{Br}_{0}(U)$ is called the algebraic part of the Brauer group. The effects of algebraic Brauer classes towards the Brauer-Manin obstruction are called the algebraic Brauer-Manin obstruction.

iii) The subgroup $\operatorname{Br}_{1}(U)$ is nothing but the kernel of the natural homomorphism $\operatorname{Br}(U) \rightarrow \operatorname{Br}\left(U_{\bar{k}}\right)$. Thus, there is a natural injection

$$
\operatorname{Br}(U) / \operatorname{Br}_{1}(U) \hookrightarrow \operatorname{Br}\left(U_{\bar{k}}\right)^{\operatorname{Gal}(\bar{k} / k)}
$$


It seems to be hard, even in concrete cases, to decide which Galois invariant Brauer classes on $U_{\bar{k}}$ actually descend to $U$. For interesting particular results, the reader is advised to study the paper [CS] of J.-L. Colliot-Thélène and A. N. Skorobogatov.

The quotient $\operatorname{Br}(U) / \operatorname{Br}_{1}(U)$ is called the transcendental part of the Brauer group. The effects of transcendental Brauer classes towards the Brauer-Manin obstruction are called the transcendental Brauer-Manin obstruction.

The algebraic part of the Brauer group.

4.4. Let $k$ be a number field, $X \subset \mathbf{P}_{k}^{4}$ a degree four del Pezzo surface, $H \subset \mathbf{P}_{k}^{4}$ a $k$-rational hyperplane such that $H \cap X$ is geometrically irreducible, and $U:=X \backslash H$. Then, $(H \cap X)^{2}=4$. Hence, by Lemma 4.1 and 4.3.ii),

$$
\operatorname{Br}_{1}(U) / \operatorname{Br}_{0}(U) \cong H^{1}\left(\operatorname{Gal}(\bar{k} / k), \operatorname{Pic}\left(U_{\bar{k}}\right)\right) .
$$

Moreover, by Lemma 3.3.ii), $\operatorname{Pic}\left(U_{\bar{k}}\right) \cong D_{5}^{*}$ and, under this isomorphism, the operation of $\operatorname{Gal}(\bar{k} / k)$ goes over into a homomorphism $\operatorname{Gal}(\bar{k} / k) \longrightarrow \operatorname{Aut}\left(D_{5}^{*}\right)$ factoring via $W\left(D_{5}\right)$.

4.5. $W\left(D_{5}\right)$ has 197 conjugacy classes of subgroups. It is known that each of them occurs already for a degree four del Pezzo surface defined over $\mathbb{Q}$. Indeed, this was shown by B. È. Kunyavskij, A. N. Skorobogatov, and M. A. Tsfasman [KST]. The reader might want to compare [EJ], which reports on a more recent investigation.

We calculated $H^{1}\left(H, D_{5}^{*}\right)$ for each conjugacy class of subgroups in $W\left(D_{5}\right)$, using magma's functionality for the computation of the cohomology of finite groups. We partially reproduce the resulting list below in Figure 1.

Remarks 4.6 (Some details on the implementation).

i) It should not be difficult to create the $\mathbb{Z} W\left(D_{5}\right)$-module $D_{5}^{*}$ within a computer algebra system. To be concrete, in magma, the command

w_d5 := TransitiveGroup $(16,1328)$;

creates the image of $W\left(D_{5}\right)$ in $S_{16}$. Then the command

subs2 := Subsets (Set (GSet (w_d5)), 2);

shows the decomposition of $\{1, \ldots, 16\} \times\{1, \ldots, 16\}$ into exactly three orbits under the $W\left(D_{5}\right)$-operation, from which the intersection matrix is easily constructed, cf. [JL, §3.1]. On the other hand, the command

Div := PermutationModule(wd5, Integers());

creates a $\mathbb{Z} W\left(D_{5}\right)$-module Div that, as a $\mathbb{Z}$-module, is free over $\{1, \ldots, 16\}$ and carries the operation of $W\left(D_{5}\right)$ that is induced by the one on that set. Thus, $D_{5}^{*} \cong \operatorname{Div} / \operatorname{Div}_{0}$, for $\operatorname{Div}_{0} \subset \operatorname{Div}$ the submodule of rank 11 given by the condition that $\left\langle x, e_{1}\right\rangle=\ldots=\left\langle x, e_{16}\right\rangle$. Here, $\langle.,$.$\rangle denotes the pairing encoded by the$ intersection matrix.

ii) As our version of magma is unwilling to compute the quotient of two $\mathbb{Z} W\left(D_{5}\right)$-modules and allows the user to do this only for $k W\left(D_{5}\right)$-modules, for $k$ 
a field, we worked modulo a large prime and recovered the structure of the $\mathbb{Z} W\left(D_{5}\right)$-module $D_{5}^{*}$ from this. It turned out that the image of $W\left(D_{5}\right)$ in $\mathrm{GL}_{5}(\mathbb{Z})$ is generated by the two matrices

$$
\left(\begin{array}{rrrrr}
1 & 0 & -1 & 0 & 1 \\
0 & 0 & 0 & 1 & 0 \\
-1 & -1 & 0 & -1 & 0 \\
0 & 0 & 1 & 0 & 0 \\
-2 & 0 & 1 & -1 & -1
\end{array}\right) \quad \text { and } \quad\left(\begin{array}{rrrrr}
2 & 1 & 0 & 1 & 1 \\
-1 & -1 & 0 & -1 & 0 \\
0 & 1 & 1 & 0 & -1 \\
-2 & 0 & 1 & -1 & -1 \\
-1 & -1 & 0 & 0 & -1
\end{array}\right) \text {. }
$$

FiguRE 1. List of algebraic Brauer groups

$1,1,[1,1,1,1,1,1,1,1,1,1,1,1,1,1,1,1],[]$,

[]

$2,2,[2,2,2,2,2,2,2,2],[2]$,

$[2,2,2]$

$3,2,[2,2,2,2,2,2,2,2],[2]$,

[ 2 ]

$4,2,[1,1,1,1,1,1,1,1,2,2,2,2],[2]$,

[]

$5,2,[1,1,1,1,2,2,2,2,2,2],[2]$,

$6,2,[2,2,2,2,2,2,2,2],[2]$,

[ 2 ]

$7,3,[1,1,1,1,3,3,3,3],[3]$,

[]

$8,5,[1,5,5,5],[5]$,

[]

$9,4,[4,4,4,4],[2,2]$,

$[2,2,2,2]$

$10,4,[2,2,2,2,2,2,2,2],[2,2]$,

[]

$11,4,[4,4,4,4],[4]$,

$[2,2,4]$

$181,96,[16],[2,2,2]$,

$[2,2]$

$189,128,[16],[2,2,2]$,

[ 2 ]

$190,192,[16],[6]$,

[ 2 ]

$191,192,[16],[2]$,

[ 2 ]

$192,192,[8,8],[2]$,

[]

$193,192,[16],[2,2,2]$,

[ 2 ]

$194,320,[16],[4]$,

[]

195, 384, [ 16$],[2,2]$,

[ 2 ]

$196,960,[16],[]$,

[]

$197,1920,[16],[2]$,

[] 
The list shows in every second line, in this order, the number of the conjugacy class in the numbering chosen by magma, the order of the corresponding subgroups, the orbit type on the 16 lines the subgroups yield, and the type of the abelian quotient. The resulting group cohomology group is indicated on the following line.

Remarks 4.7. i) To summarise, we find that $H^{1}\left(H, D_{5}^{*}\right)$ is

- 0 in 59 cases,

- $\mathbb{Z} / 2 \mathbb{Z}$ in 62 cases,

- $(\mathbb{Z} / 2 \mathbb{Z})^{2}$ in 44 cases,

- $(\mathbb{Z} / 2 \mathbb{Z})^{3}$ in 16 cases,

- $(\mathbb{Z} / 2 \mathbb{Z})^{4}$ in three cases,

- $\mathbb{Z} / 4 \mathbb{Z}$ in nine cases,

- $\mathbb{Z} / 2 \mathbb{Z} \times \mathbb{Z} / 4 \mathbb{Z}$ in three cases, and

- $(\mathbb{Z} / 2 \mathbb{Z})^{2} \times \mathbb{Z} / 4 \mathbb{Z}$ in one case.

In comparison with the Brauer groups of proper degree four del Pezzo surfaces, which may be only $0, \mathbb{Z} / 2 \mathbb{Z}$, or $(\mathbb{Z} / 2 \mathbb{Z})^{2}$ [Ma, Section 31, Table 3] (see also [SD]), these figures are unexpectedly rich.

ii) The running time was a little less than one second on one core of a AMD Phenom II X4 955 processor, using magma, version 2.20.8.

iii) Note that Manin's formula [Ma, Proposition 31.3] (see also [Ja, Chapter III, Proposition 8.18]) does not apply to the computation of $H^{1}\left(H, D_{5}^{*}\right)$, as $D_{5}^{*}$ is not a unimodular lattice.

In the theorem below, we list some cases of particular interest.

Theorem 4.8. Let $k$ be a number field, $X \subset \mathbf{P}_{k}^{4}$ a degree four del Pezzo surface, $H \subset \mathbf{P}_{k}^{4}$ be a k-rational hyperplane such that $H \cap X$ is geometrically irreducible, and put $U:=X \backslash H$.

a) Then $\operatorname{Br}_{1}(U) / \operatorname{Br}_{0}(U)$ is isomorphic to $0, \mathbb{Z} / 2 \mathbb{Z},(\mathbb{Z} / 2 \mathbb{Z})^{2},(\mathbb{Z} / 2 \mathbb{Z})^{3},(\mathbb{Z} / 2 \mathbb{Z})^{4}$, $\mathbb{Z} / 4 \mathbb{Z}, \mathbb{Z} / 2 \mathbb{Z} \times \mathbb{Z} / 4 \mathbb{Z}$, or $(\mathbb{Z} / 2 \mathbb{Z})^{2} \times \mathbb{Z} / 4 \mathbb{Z}$.

b) Suppose that

i) the Galois group faithfully operating on the 16 lines on $X$ is isomorphic to the full $W\left(D_{5}\right)$ or its index two subgroup. Then $\operatorname{Br}_{1}(U) / \operatorname{Br}_{0}(U)=0$.

ii) the Galois group faithfully operating on the 16 lines on $X$ is isomorphic to the index five subgroup in $W\left(D_{5}\right)$. Then $\operatorname{Br}_{1}(U) / \mathrm{Br}_{0}(U)=\mathbb{Z} / 2 \mathbb{Z}$.

iii) two of the five degenerate quadrics in the pencil associated with $X$ are defined over $k$ and the Galois group faithfully operating on the 16 lines on $X$ is of index 20 in $W\left(D_{5}\right)$. Then $\operatorname{Br}_{1}(U) / \operatorname{Br}_{0}(U)=(\mathbb{Z} / 2 \mathbb{Z})^{2}$.

Proof. a), b.i) and b.ii) are immediate consequences from the list above.

b.iii) According to Facts 3.1.iii and v), there is a canonical isomorphism

$$
W\left(D_{5}\right) \longrightarrow(\mathbb{Z} / 2 \mathbb{Z})^{4} \rtimes S_{5},
$$


where the factor $S_{5}$ is the group permuting the five degenerate quadrics in the pencil associated with $X$. Thus, by our first assumption, the Galois group $H \hookrightarrow W\left(D_{5}\right)$ faithfully operating on the 16 lines is mapped isomorphically onto a subgroup of $(\mathbb{Z} / 2 \mathbb{Z})^{4} \rtimes S_{3} \subset(\mathbb{Z} / 2 \mathbb{Z})^{4} \rtimes S_{5}$, which is of order 96 . On the other hand, order 96 is assumed, so that $H$ goes over exactly into that semidirect product. It is not hard to check that 181 is the corresponding number in the list.

The transcendental part of the Brauer group.

Theorem 4.9. Let $k$ be an algebraically closed field of characteristic 0 and $U:=X \backslash H$, for $X \subset \mathbf{P}_{k}^{4}$ a degree four del Pezzo surface and $H \subset \mathbf{P}_{k}^{4}$ a hyperplane such that $D:=H \cap X$ is non-singular. Then there is a canonical isomorphism

$$
\operatorname{Br}(U) \cong H_{\text {ét }}^{1}(D, \mathbb{Q} / \mathbb{Z}) \text {. }
$$

Proof. Let $n$ be any positive integer. Then, the Gysin sequence [SGA5, Exp. VII, Corollaire 1.5] (or [Mi, Chapter VI, Remark 5.4.b)]) reads

$$
H_{\text {ét }}^{0}(D, \mathbb{Z} / n \mathbb{Z}) \longrightarrow H_{\text {ét }}^{2}\left(X, \mu_{n}\right) \longrightarrow H_{\text {ét }}^{2}\left(U, \mu_{n}\right) \longrightarrow H_{\text {ét }}^{1}(D, \mathbb{Z} / n \mathbb{Z}) \longrightarrow H_{\text {ét }}^{3}\left(X, \mu_{n}\right) \text {. }
$$

Here, as $X$ is a del Pezzo surface, one has $H_{\text {ét }}^{2}\left(X, \mu_{n}\right) \cong \operatorname{Pic}(X) / n \operatorname{Pic}(X)$ and $H_{\text {ét }}^{3}\left(X, \mu_{n}\right)=0$. Moreover, $H_{\text {ét }}^{0}(D, \mathbb{Z} / n \mathbb{Z})=\mathbb{Z} / n \mathbb{Z}$ and the generator is mapped to the anticanonical class in $\operatorname{Pic}(X) / n \operatorname{Pic}(X)$. Therefore, in view of [Hart, Proposition II.6.5.c)], the sequence simplifies to

$$
0 \longrightarrow \operatorname{Pic}(U) / n \operatorname{Pic}(U) \longrightarrow H_{\text {èt }}^{2}\left(U, \mu_{n}\right) \longrightarrow H_{\text {ét }}^{1}(D, \mathbb{Z} / n \mathbb{Z}) \longrightarrow 0,
$$

which shows that $\operatorname{Br}(U)_{n} \cong H_{\text {ét }}^{1}(D, \mathbb{Z} / n \mathbb{Z})$. As $n$ was arbitrary, this implies that $\operatorname{Br}(U)_{\text {tors }} \cong H_{\text {ét }}^{1}(D, \mathbb{Q} / \mathbb{Z})$.

However, for non-singular schemes in general, it is known that the cohomological Brauer group is torsion [GrBrII, Proposition 1.4].

Corollary 4.10. Let $k$ be a number field and $U:=X \backslash H$, for $X \subset \mathbf{P}_{k}^{4}$ a degree four del Pezzo surface and $H \subset \mathbf{P}_{k}^{4}$ a $k$-rational hyperplane such that $D:=H \cap X$ is non-singular. Then, for every $n \geq 2$, there is a canonical monomorphism

$$
\left[\operatorname{Br}(U) / \operatorname{Br}_{1}(U)\right]_{n} \hookrightarrow\left(J(D)(\bar{k})_{n} \otimes_{\mathbb{Z} / n \mathbb{Z}} \mu_{n}^{\vee}\right)^{\operatorname{Gal}(\bar{k} / k)}
$$

for $J(D)$ the Jacobian variety of $D$.

Proof. By 4.3.iii), there is a canonical injection $\operatorname{Br}(U) / \operatorname{Br}_{1}(U) \hookrightarrow \operatorname{Br}\left(U_{\bar{k}}\right)^{\operatorname{Gal}(\bar{k} / k)}$. Moreover, Theorem 4.9 yields a canonical isomorphism

$$
\operatorname{Br}\left(U_{\bar{k}}\right)_{n} \cong H_{\text {ét }}^{1}\left(D_{\bar{k}}, \mathbb{Z} / n \mathbb{Z}\right) \cong H_{\text {ét }}^{1}\left(D_{\bar{k}}, \mu_{n}\right) \otimes_{\mathbb{Z} / n \mathbb{Z}} \mu_{n}^{\vee}
$$

Here, $H_{\text {ét }}^{1}\left(D_{\bar{k}}, \mu_{n}\right) \cong \operatorname{Pic}\left(D_{\bar{k}}\right)_{n}$ by virtue of [Mi, Chapter III, Proposition 4.11]. Moreover, as $D$ is a non-singular curve, one has $\operatorname{Pic}\left(D_{\bar{k}}\right)_{n} \cong J(D)(\bar{k})_{n}$, according to the Picard interpretation of the Jacobian. 
Remarks 4.11. i) For the rest of the paper, we only consider 2-torsion classes. In this case, the inclusion (3) simplifies to

$$
\left[\operatorname{Br}(U) / \operatorname{Br}_{1}(U)\right]_{2} \hookrightarrow J(D)(k)_{2} .
$$

Moreover, if $D$ has a $k$-rational point $O$ then we may choose this point to convert $D$ into an elliptic curve and get an injection into the $k$-rational 2 -torsion points of the elliptic curve $D$.

ii) As indicated for a more general situation in paragraph 4.3.iii), it may well be a hard problem to decide which elements on the right hand side of (3) belong to the image of this monomorphism. In some particular cases, however, we are able to write down transcendental Brauer classes, explicitly. Cf. Theorem 5.5 below.

iii) By Poincaré duality [Mi, Chapter VI, Corollary 11.2], we have a perfect, Galoisinvariant paring

$$
H_{\text {ét }}^{1}\left(D_{\bar{k}}, \mu_{n}\right) \times H_{\text {ét }}^{1}\left(D_{\bar{k}}, \mathbb{Z} / n \mathbb{Z}\right) \rightarrow H_{\text {ét }}^{2}\left(D_{\bar{k}}, \mu_{n}\right) \cong \mathbb{Z} / n \mathbb{Z},
$$

which induces an isomorphism

$$
H_{\text {ét }}^{1}\left(D_{\bar{k}}, \frac{1}{n} \mathbb{Z} / \mathbb{Z}\right) \cong \operatorname{Hom}\left(H_{\text {ét }}^{1}\left(D_{\bar{k}}, \mu_{n}\right), \frac{1}{n} \mathbb{Z} / \mathbb{Z}\right) \cong \operatorname{Hom}\left(J(D)(\bar{k})_{n}, \mathbb{Q} / \mathbb{Z}\right) .
$$

Hence one obtains, similarly as in Corollary 4.10, a monomorphism

$$
\left[\operatorname{Br}(U) / \operatorname{Br}_{1}(U)\right]_{n} \hookrightarrow \operatorname{Hom}_{\operatorname{Gal}(\bar{k} / k)}\left(J(D)(\bar{k})_{n}, \mathbb{Q} / \mathbb{Z}\right)
$$

In particular, a proper $n$-torsion class in $\operatorname{Br}(U) / \operatorname{Br}_{1}(U)$ causes a $k$-rational $n$-isogeny $J(D) \rightarrow D^{\prime}$ to an elliptic curve $D^{\prime}$ with a $k$-rational proper $n$-torsion point.

\section{Explicit BRAUER CLASSES}

The tame symbol.

Let $X$ be an irreducible, non-singular scheme of dimension $\leq 2$ over a field $k$ of characteristic 0. Then there is a canonical monomorphism $\operatorname{Br}(X) \hookrightarrow \operatorname{Br}(k(X))$. Moreover, within $\operatorname{Br}(k(X))$, one has [GrBrII, Proposition 2.3]

$$
\operatorname{Br}(X) \cong \bigcap_{x \in X^{(1)}} \operatorname{Br}\left(\operatorname{Spec} \mathscr{O}_{X, x}\right)
$$

where $X^{(1)}$ denotes the set of all codimension-one points on the scheme $X$.

In other words, a Brauer class of the function field $k(X)$ extends to the whole of $X$ if and only if it is unobstructed at any prime divisor. And if this is the case then the extension is unique.

The obstruction at a prime divisor $x$ is tested by the so-called ramification homomorphism. This is the following composition [GrBrIII, Proposition 2.1],

$$
\begin{array}{r}
\operatorname{ram}_{x}: \operatorname{Br}(k(X)) \stackrel{\cong}{\longleftarrow} H_{\text {ét }}^{2}\left(X, \mathbb{G}_{m, k(X)}\right) \longrightarrow H_{\text {ét }}^{2}\left(X, i_{*} \mathbb{Z}_{x}\right) \stackrel{\cong}{\longleftarrow} H_{\text {ét }}^{1}(\operatorname{Spec} k(x), \mathbb{Q} / \mathbb{Z}) \\
=H^{1}(\operatorname{Gal}(\overline{k(x)} / k(x)), \mathbb{Q} / \mathbb{Z}) .
\end{array}
$$


Here, the first map is an isomorphism due to [Ja, Chapter III, Corollary 5.6], the second is the homomorphism induced by the valuation map

$$
\operatorname{val}_{x}: \mathbb{G}_{m, k(X)} \rightarrow i_{*} \mathbb{Z}_{x}
$$

and the third is the connecting homomorphism induced by the short exact sequence $0 \rightarrow \mathbb{Z} \rightarrow \mathbb{Q} \rightarrow \mathbb{Q} / \mathbb{Z} \rightarrow 0$.

A way to explicitly write down a Brauer class of the function field is provided by the cyclic algebras. For these, let $n \in \mathbb{N}$ and suppose that $k(X)$ contains a primitive $n$-th root of unity $\zeta_{n}$. Then, for $f, g \in k(X)^{*}$,

$$
\left(f, g ; \zeta_{n}\right):=\bigoplus_{0 \leq a, b<n} u^{a} v^{b} k(X) \quad \text { for } u^{n}=f, v^{n}=g, v u=u v \zeta_{n},
$$

is an Azumaya algebra [Pi, §15.4, Proposition] and therefore defines a class in $\operatorname{Br}(k(X))$. The corresponding cohomology class in $\left.H^{2}(\operatorname{Gal}(\overline{k(X)} / k(X)), \overline{k(X)})^{*}\right)$ may be described by the cocycle $\Phi$ such that [Pi, $\S 15.1$, Proposition a]

$$
\Phi(\sigma, \tau)= \begin{cases}1 & \text { if } i+j<n, \\ f & \text { if } i+j \geq n,\end{cases}
$$

for $0 \leq i, j<n$ chosen by the condition that $\sigma(\sqrt[n]{g})=\zeta_{n}^{i} \sqrt[n]{g}$ and $\tau(\sqrt[n]{g})=\zeta_{n}^{j} \sqrt[n]{g}$.

Finally, recall that there is the tame symbol

$$
\delta_{x}: k(X)^{*} \times k(X)^{*} \longrightarrow k(x)^{*}, \quad(f, g) \mapsto(-1)^{\nu_{x}(f) \nu_{x}(g)} \overline{\left(\frac{f^{\nu_{x}(g)}}{g^{\nu_{x}(f)}}\right)},
$$

where the bar denotes the natural residue map $\mathscr{O}_{X, x} \rightarrow k(x)$.

Proposition 5.1. Let $n \in \mathbb{N}$ and fix a primitive $n$-th root of unity $\zeta_{n} \in k(X)^{*}$. Then the following diagram commutes,

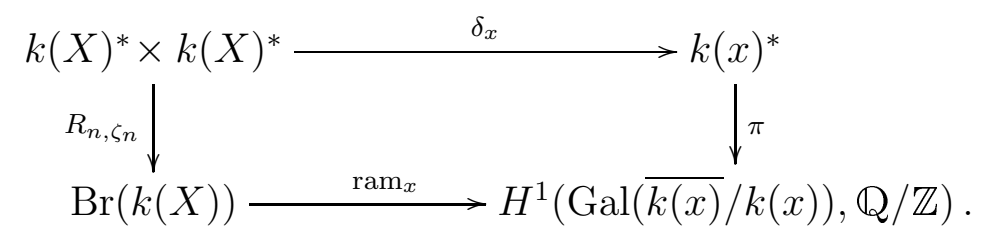

Here, the downward arrow $R_{n, \zeta_{n}}$ to the left sends $(f, g)$ to the Brauer class of $\left(f, g ; \zeta_{n}\right)$, while the one to the right is the canonical map

$$
k(x)^{*} \rightarrow k(x)^{*} /\left(k(x)^{*}\right)^{n} \stackrel{\cong}{\longrightarrow} H^{1}\left(\mathrm{Gal}(\overline{k(x)} / k(x)), \mu_{n}\right),
$$

followed by the isomorphism induced by the identification $\mu_{n} \rightarrow \frac{1}{n} \mathbb{Z} / \mathbb{Z}, \zeta_{n} \mapsto-\frac{1}{n}$.

Proof. If $\nu_{x}(f)=\nu_{x}(g)=0$ then $\delta_{x}(f, g)=1$ such that $(f, g)$ is mapped to 0 via the upper right corner.

On the other hand, the cohomology class of $R_{n, \zeta_{n}}(f, g)=\left(f, g ; \zeta_{n}\right)$ comes via inflation from $H^{2}\left(\operatorname{Gal}(k(X)(\sqrt[n]{g}) / k(X)), \overline{k(X)^{*}}\right)$ and is represented by a cocycle whose image is $\{1, f\}$. Since the field extension $k(X)(\sqrt[n]{g}) / k(X)$ is unramified at $x$ and one has $\nu_{x}(1)=\nu_{x}(f)=0$, it follows that $\operatorname{ram}_{x}\left(f, g ; \zeta_{n}\right)$ is represented by the 
cocycle that is constantly zero. Hence, $\operatorname{ram}_{x}\left(f, g ; \zeta_{n}\right)=0$. Thus, via the lower left corner, $(f, g)$ is mapped to 0 , too.

Next, we observe that the maps $\delta_{x}$ and $R_{n, \zeta_{n}}$ are bimultiplicative and antisymmetric. Thus, in order to complete the proof, it suffices to consider the case that $\nu_{x}(f)=1$ and $\nu_{x}(g)=0$.

Upper right path: We have $\delta_{x}(f, g)=\overline{(1 / g)}$, which is mapped under $\pi$ to the class of the homomorphism

$$
\sigma \mapsto \frac{\sigma(\sqrt[n]{\overline{(1 / g)}})}{\sqrt[n]{\overline{(1 / g)}})}=\frac{\sqrt[n]{\bar{g}}}{\sigma(\sqrt[n]{\bar{g}})}
$$

in $H^{1}\left(\operatorname{Gal}(k(x)(\sqrt[n]{\bar{g}}) / k(x)), \mu_{n}\right) \hookrightarrow H^{1}\left(\operatorname{Gal}(\overline{k(x)} / k(x)), \mu_{n}\right)$. In other words, $\pi\left(\delta_{x}(f, g)\right)$ maps the element $\sigma \in \operatorname{Gal}(k(x)(\sqrt[n]{\bar{g}}) / k(x))$ such that $\sqrt[n]{\bar{g}} \mapsto \zeta_{n}^{i} \sqrt[n]{\bar{g}}$ to $\zeta_{n}^{-i}$, which finally gets identified with $\frac{i}{n}$.

Lower left path: Here, $(f, g)$ is sent first by $R_{n, \zeta_{n}}$ to $\left(f, g ; \zeta_{n}\right)$, which is described by the cocycle $\Phi$ such that

$$
\Phi(\sigma, \tau)= \begin{cases}1 & \text { if } i+j<n \\ f & \text { if } i+j \geq n\end{cases}
$$

for $0 \leq i, j<n$ chosen in the way that $\sigma(\sqrt[n]{g})=\zeta_{n}^{i} \sqrt[n]{g}$ and $\tau(\sqrt[n]{g})=\zeta_{n}^{j} \sqrt[n]{g}$. Applying the valuation $\nu_{x}$, one finds

$$
\left(\nu_{x} \circ \Phi\right)(\sigma, \tau)= \begin{cases}0 & \text { if } i+j<n, \\ 1 & \text { if } i+j \geq n .\end{cases}
$$

This, however, is exactly the image of

$$
\Psi: \sigma \mapsto \frac{i}{n}, \quad \text { for } \sigma(\sqrt[n]{g})=\zeta_{n}^{i} \sqrt[n]{g}
$$

understood as an element in $H^{1}(\operatorname{Gal}(k(X)(\sqrt[n]{g}) / k(X)), \mathbb{Q} / \mathbb{Z})$, under the connecting homomorphism associated with $0 \rightarrow \mathbb{Z} \rightarrow \mathbb{Q} \rightarrow \mathbb{Q} / \mathbb{Z} \rightarrow 0$. Thus, $\operatorname{ram}_{x}\left(R_{n, \zeta_{n}}(f, g)\right)$ maps the element $\sigma \in \operatorname{Gal}(k(x)(\sqrt[n]{\bar{g}}) / k(x))$ sending $\sqrt[n]{\bar{g}} \mapsto \zeta_{n}^{i} \sqrt[n]{\bar{g}}$ to $\frac{i}{n}$. This completes the proof.

Corollary 5.2. Let $X$ be an irreducible, non-singular scheme of dimension $\leq 2$ over a field $k$ of characteristic $0, n \in \mathbb{N}, \zeta_{n} \in k(X)$ a primitive $n$-th root of unity, $f, g \in k(X)^{*}$, and $x \in X^{(1)}$ a prime divisor. Then the Brauer class of $\left(f, g ; \zeta_{n}\right)$ is unobstructed at $x$ if and only if $\delta_{x}(f, g)$ is an $n$-th power in $k(x)$.

Remarks 5.3. i) In Theorem 4.9, the Gysin homomorphism appeared instead of the tame symbol. It is, in fact, true that these two maps are compatible. Cf., for example, [BMT, Lemma 2.5].

ii) In [AM, §3], M. Artin and D. Mumford provide a more complete picture of the ramification homomorphism. For example, the following is shown.

Let $X$ be a proper surface over an algebraically closed field and $U:=X \backslash D$ for $D$ an irreducible non-singular curve. Suppose that $\left(f, g ; \zeta_{n}\right)$ defines a Brauer class on $U$. 
Then the divisor div $t$ of the rational function $t:=\delta_{x}(f, g) \in k(D)$, for $x$ the generic point of $D$, is divisible by $n$.

Of course, on a curve of genus $\geq 1$, this is not enough to imply that $t$ has to be an $n$-th power.

Two model cases.

Theorem 5.4 (An algebraic Brauer class). Let $k$ be any field of characteristic $0, X \subset \mathbf{P}_{k}^{4}$ a del Pezzo surface of degree four over $k$, and $U:=X \backslash H$ for $H:=V\left(X_{4}\right) \subset \mathbf{P}_{k}^{4}$. Suppose that the pencil of quadrics associated with $X$ contains a k-rational quadric of rank 4 that may be written in the form

$$
l_{1} l_{2}-l_{3}^{2}+d l_{4}^{2},
$$

for linear forms $l_{1}, \ldots, l_{4}$ and $d \in k^{*}$ a non-square.

i) Then

$$
\left(\frac{l_{1}}{X_{4}}, d ;-1\right)
$$

defines an algebraic Brauer class $\alpha \in \operatorname{Br}_{1}(U)_{2}$.

ii) If $V\left(X_{4}\right) \cap X$ is reduced and has a $k$-rational point then $\alpha$ does not extend to $X$ and is, in particular, nontrivial.

Proof. i) Let $D \subset U$ be any prime divisor and $x \in U^{(1)}$ its generic point. Then $\nu_{x}(d)=0$ and $\nu_{x}\left(\frac{l_{1}}{X_{4}}\right)=0$, unless $D$ is a component of $V\left(l_{1}\right) \cap X$. Thus, if $D \not \subset V\left(l_{1}\right) \cap X$ then $\delta_{x}\left(\frac{l_{1}}{X_{4}}, d\right)=1$.

On the other hand, if $D \subset V\left(l_{1}\right) \cap X$ then $\delta_{x}\left(\frac{l_{1}}{X_{4}}, d\right)=\frac{1}{d^{\nu x\left(l_{1}\right)}}$, which is a square on $D$, since the equation $-l_{3}^{2}+d l_{4}^{2}=0$ implies

$$
d=\frac{l_{3}^{2}}{l_{4}^{2}} .
$$

The Brauer class $\alpha$ is clearly algebraic, as it gets annihilated under base extension to $k(\sqrt{d})$.

ii) Take now $D$ to be a component of $V\left(X_{4}\right) \cap X$. Then $\delta_{x}\left(\frac{l_{1}}{X_{4}}, d\right)=d$ for $x \in U^{(1)}$ the generic point of $D$, which cannot be a square when $D$ has a $k$-rational point.

Theorem 5.5 (A transcendental Brauer class). Let $k$ be any field of characteristic 0 and $X \subset \mathbf{P}_{k}^{4}$ a del Pezzo surface of degree four over $k$ that is given by a system of equations of the type

$$
\begin{aligned}
& l_{1} l_{2}+a u^{2}=X_{4} l_{3}, \\
& l_{3} l_{4}+b v^{2}=X_{4} l_{1},
\end{aligned}
$$

for linear forms $l_{1}, \ldots, l_{4}, u, v$, and $a, b \in k^{*}$. Assume that the forms $l_{1}, l_{3}, u$, and $v$ are linearly independent.

Put $U:=X \backslash H$ for $H:=V\left(X_{4}\right) \subset \mathbf{P}_{k}^{4}$.

i) Then

$$
\left(\frac{b l_{1}}{X_{4}}, \frac{a l_{3}}{X_{4}} ;-1\right)
$$

defines a Brauer class $\tau \in \operatorname{Br}(U)_{2}$. 
ii) If $D:=V\left(X_{4}\right) \cap X$ is geometrically integral and $\frac{l_{1}}{l_{3}}$ is not the square of a rational function on $D_{\bar{k}}$ then $\tau_{U_{\bar{k}}}$ does not extend to $X_{\bar{k}}$. In particular, $\tau$ is transcendental.

Proof. i) Let $D \subset U$ be any prime divisor and $x \in U^{(1)}$ its generic point. Then $\nu_{x}\left(\frac{b l_{1}}{X_{4}}\right)=0$ and $\nu_{x}\left(\frac{a l_{3}}{X_{4}}\right)=0$, unless $D \subset V\left(l_{1}\right) \cap X$ or $D \subset V\left(l_{3}\right) \cap X$. Thus, if $D$ is not a component of either of these two subschemes then $\delta_{x}\left(\frac{b l_{1}}{X_{4}}, \frac{a l_{3}}{X_{4}}\right)=1$.

On the other hand, if $D \subset V\left(l_{1}\right) \cap X$, but $D \not \subset V\left(l_{3}\right) \cap X$, then one finds that $\delta_{x}\left(\frac{b l_{1}}{X_{4}}, \frac{a l_{3}}{X_{4}}\right)=\left(\frac{X_{4}}{a l_{3}}\right)^{\nu_{x}\left(l_{1}\right)}$, which is a square on $D$, since the equation $a u^{2}=X_{4} l_{3}$ implies

$$
\frac{X_{4}}{a l_{3}}=\frac{u^{2}}{l_{3}^{2}} .
$$

Similarly, if $D \subset V\left(l_{3}\right) \cap X$, but $D \not \subset V\left(l_{1}\right) \cap X$, then $\delta_{x}\left(\frac{b l_{1}}{X_{4}}, \frac{a l_{3}}{X_{4}}\right)=\left(\frac{b l_{1}}{X_{4}}\right)^{\nu_{x}}\left(l_{3}\right)$, which is a square on $D$, since $b v^{2}=X_{4} l_{1}$ yields

$$
\frac{b l_{1}}{X_{4}}=\frac{l_{1}^{2}}{v^{2}} .
$$

Finally, there cannot be a divisor that is contained in both, $V\left(l_{1}\right) \cap X$ and $V\left(l_{3}\right) \cap X$, as this would imply $l_{1}=l_{3}=u=v=0$, which, according to our assumptions, is fulfilled only by a single point.

ii) Let now $x$ be the generic point of $V\left(X_{4}\right) \cap X$. Then $\delta_{x}\left(\frac{b l_{1}}{X_{4}}, \frac{a l_{3}}{X_{4}}\right)=\frac{a l_{3}}{b l_{1}}$. Since $\frac{a}{b}$ is a square in $\bar{k}$, this immediately implies the claim.

Constancy of the local evaluation maps.

Remark 5.6. Let now $k$ be a number field and $U$ be a $k$-scheme, on which a Brauer class $\alpha \in \operatorname{Br}(U)$ is given by $(f, g ;-1)$, for rational functions $f, g \in k(U)$. Moreover, let $\nu$ be any place of $k$ and $x \in U\left(\mathbb{Q}_{\nu}\right), x \notin \operatorname{supp} \operatorname{div} f \cup \operatorname{supp} \operatorname{div} g$, a point. Then

$$
\operatorname{ev}_{\alpha, \nu}(x)= \begin{cases}0 & \text { if }(f(x), g(x))_{\nu}=1 \\ \frac{1}{2} & \text { if }(f(x), g(x))_{\nu}=-1\end{cases}
$$

where $(., .)_{\nu}: k_{\nu}^{*} \times k_{\nu}^{*} \rightarrow\{1,-1\}$ denotes the usual $\nu$-adic Hilbert symbol.

Indeed, according to the definition, $\mathrm{ev}_{\alpha, \nu}(x)=x^{*} \alpha$ and our assumptions guarantee that the pull-back may be obtained in a naive way as the cyclic (quaternion) algebra $(f(x), g(x) ;-1)$ over $k_{\nu}$. Finally, the invariant of such an algebra is directly related, by the above rule, to the Hilbert symbol [Pi, §18.4, Exercise 4].

Lemma 5.7. Let $k$ be a number field, $\mathscr{X} \subset \mathbf{P}_{\mathscr{O}_{k}}^{4}$ the closed subscheme defined by two quadratic forms with coefficients in $\mathscr{O}_{k}$, and $\mathscr{U}:=\mathscr{X} \backslash \mathscr{H}$ for $\mathscr{H}:=V\left(X_{4}\right) \subset \mathbf{P}_{\mathscr{O}_{k}}^{4}$. Suppose that the generic fibre $X$ of $\mathscr{X}$ is a non-singular surface and let $U$ and $\alpha \in \operatorname{Br}(U)_{2}$ be as in Theorem 5.4.

Assume that one of the quadratic forms defining $\mathscr{X}$ may be written in the form

$$
l_{1} l_{2}-l_{3}^{2}+d l_{4}^{2}
$$

for $l_{1}, \ldots, l_{4}$ linear forms having coefficients in $\mathscr{O}_{k}$ and $d \in \mathscr{O}_{k}$ a non-square. Furthermore, let $\mathfrak{p}$ be a prime ideal in $\mathscr{O}_{k}$ such that $\mathfrak{p} \nmid 2 d$,

- the linear forms $\left(l_{1} \bmod \mathfrak{p}\right), \ldots,\left(l_{4} \bmod \mathfrak{p}\right)$ are $\mathscr{O}_{k} / \mathfrak{p}$-linearly independent, and 
- the reduction modulo $\mathfrak{p}$ of the cusp given by $l_{1}=\ldots=l_{4}=0$ does not lie on the reduction $\mathscr{U}_{\mathfrak{p}}$.

Then the local evaluation map

$$
\operatorname{ev}_{\alpha, \nu_{\mathfrak{p}}}: \mathscr{U}\left(\mathscr{O}_{k, \mathfrak{p}}\right) \longrightarrow \mathbb{Q} / \mathbb{Z}
$$

is constantly zero.

Proof. Let us denote integral points $x \in \mathscr{U}\left(\mathscr{O}_{k, \mathfrak{p}}\right)$ in the form $x=\left(x_{0}: \ldots: x_{3}: 1\right)$, for $x_{0}, \ldots, x_{3} \in \mathscr{O}_{k, \mathfrak{p}}$. The local evaluation map ev $\mathrm{e}_{\alpha, \nu_{\mathfrak{p}}}$ is known to be locally constant with respect to the $\mathfrak{p}$-adic topology on $U\left(k_{\nu_{\mathfrak{p}}}\right)$ [Ja, Chapter IV, Proposition 2.3.a.ii)]. Consequently, it suffices to prove the assertion for points $x \in \mathscr{U}\left(\mathscr{O}_{k, \mathfrak{p}}\right)$ such that $l_{1}(x) \neq 0$ and $l_{2}(x) \neq 0$. Thus, what we have to show is

$$
\left(l_{1}\left(x_{0}, \ldots, x_{3}, 1\right), d\right)_{\nu_{\mathfrak{p}}}=1
$$

for every point $x=\left(x_{0}: \ldots: x_{3}: 1\right) \in U\left(\mathscr{O}_{k, \mathfrak{p}}\right)$ satisfying the inequalities above.

For this, we first observe that the equation given yields $l_{1}(x) l_{2}(x)=l_{3}^{2}(x)-d l_{4}^{2}(x)$, which is a norm from $k_{\nu_{\mathfrak{p}}}(\sqrt{d})$. Therefore, in the Hilbert symbol, we may replace $l_{1}(x)$ by $l_{2}(x)$.

If $(d \bmod \mathfrak{p}) \in \mathscr{O}_{k} / \mathfrak{p}$ is a square then assertion is certainly true. Thus assume that $(d \bmod \mathfrak{p}) \in \mathscr{O}_{k} / \mathfrak{p}$ is a non-square. Then $\left(l_{1}(x), d\right)_{\nu_{\mathfrak{p}}}=-1$ would mean that $\nu_{\mathfrak{p}}\left(l_{1}(x)\right)$ is odd. In which case, $\nu_{\mathfrak{p}}\left(l_{2}(x)\right)$ is also odd. In particular, $\mathfrak{p} \mid l_{1}(x)$ and $\mathfrak{p} \mid l_{2}(x)$, which directly implies that $\mathfrak{p} \mid l_{3}(x)$ and $\mathfrak{p} \mid l_{4}(x)$, too.

However, according to our assumptions, such an $\mathscr{O}_{k, \mathfrak{p}}$-valued point on $\mathscr{U}$ does not exist. Indeed, its reduction modulo $\mathfrak{p}$ would coincide with that of the cusp, which is supposed not to lie on $\mathscr{U}_{\mathfrak{p}}$.

Remark 5.8. In particular, we see that if $\mathfrak{p} \nmid 2$ is a prime ideal of good reduction of $\mathscr{X}$ then $\mathrm{ev}_{\alpha, \mathfrak{p}}$ is constantly zero.

Indeed, then $\mathscr{X}_{\mathfrak{p}}$ is a degree four del Pezzo surface over $\mathbb{F}_{\mathfrak{p}}$. Hence, the rank of a degenerate quadric in the pencil cannot drop below 4 [Wi, Proposition 3.26.iv)]. This implies immediately that $\mathfrak{p} \nmid d$, as well as linear independence of $\left(l_{1} \bmod \mathfrak{p}\right), \ldots,\left(l_{4} \bmod \mathfrak{p}\right)$. Moreover, the reduction of the cusp would be a singular point on $\mathscr{U}_{\mathfrak{p}}$, which, by assumption, does not exist.

Lemma 5.9. Let $k$ be a number field and $\mathscr{X} \subset \mathbf{P}_{\mathscr{O}_{k}}^{4}$ be the closed subscheme defined by a system of equations of the type

$$
\begin{aligned}
& l_{1} l_{2}+a u^{2}=X_{4} l_{3}, \\
& l_{3} l_{4}+b v^{2}=X_{4} l_{1},
\end{aligned}
$$

for linear forms $l_{1}, \ldots, l_{4}, u, v$ having coefficients in $\mathscr{O}_{k}$ and $a, b \in \mathscr{O}_{k}^{*}$. Assume that the forms $l_{1}, l_{3}, u$, and $v$ are $k$-linearly independent and that the generic fibre $X$ of $\mathscr{X}$ is a non-singular surface.

Put $\mathscr{U}:=\mathscr{X} \backslash \mathscr{H}$ for $\mathscr{H}:=V\left(X_{4}\right) \subset \mathbf{P}_{\mathscr{O}_{k}}^{4}$ and let $U$ and $\tau \in \operatorname{Br}(U)_{2}$ be as in Theorem 5.5. Then, for every prime ideal $\mathfrak{p}$ in $\mathscr{O}_{k}$ such that $\mathfrak{p} \nmid 2 a b$, the local 
evaluation map

$$
\mathrm{ev}_{\tau, \nu_{\mathfrak{p}}}: \mathscr{U}\left(\mathscr{O}_{k, \mathfrak{p}}\right) \longrightarrow \mathbb{Q} / \mathbb{Z}
$$

is constantly zero.

Proof. Let us denote integral points $x \in \mathscr{U}\left(\mathscr{O}_{k, \mathfrak{p}}\right)$ in the form $x=\left(x_{0}: \ldots: x_{3}: 1\right)$ for $x_{0}, \ldots, x_{3} \in \mathscr{O}_{k, \mathfrak{p}}$. As the local evaluation map $\mathrm{ev}_{\tau, \nu_{\mathfrak{p}}}$ is locally constant with respect to the $\mathfrak{p}$-adic topology on $U\left(k_{\nu_{\mathfrak{p}}}\right)$, it suffices to prove the assertion for points $x \in \mathscr{U}\left(\mathscr{O}_{k, \mathfrak{p}}\right)$ such that $l_{i}(x) \neq 0$, for $i=1, \ldots, 4$. Thus, what we have to show is

$$
\left(b l_{1}(x), a l_{3}(x)\right)_{\nu_{\mathfrak{p}}}=1
$$

for every point $x=\left(x_{0}: \ldots: x_{3}: 1\right) \in \mathscr{U}\left(\mathscr{O}_{k, \mathfrak{p}}\right)$ satisfying the inequalities above.

First of all, observe that $a$ and $b$ are $\mathfrak{p}$-adic units. Furthermore, the first equation yields $(-a) l_{1}(x) l_{2}(x)=a^{2} u^{2}(x)-a l_{3}(x)$, which is a norm from $k_{\nu_{\mathfrak{p}}}\left(\sqrt{a l_{3}(x)}\right)$. Therefore, in the Hilbert symbol, we may replace $b l_{1}(x)$ by $(-a b) l_{2}(x)$.

Analogously, we have $(-b) l_{3}(x) l_{4}(x)=b^{2} v^{2}(x)-b l_{1}(x)$ being a norm from $k_{\nu_{\mathfrak{p}}}\left(\sqrt{b l_{1}(x)}\right)$. Thus, we may as well replace $a l_{3}(x)$ by $(-a b) l_{4}(x)$.

This shows, if one of the numbers $l_{1}(x), l_{2}(x)$ is of even $\mathfrak{p}$-adic valuation, as well as one of the numbers $l_{3}(x), l_{4}(x)$, then assertion is certainly true. Thus, suppose without restriction that $\nu_{\mathfrak{p}}\left(l_{1}(x)\right)$ and $\nu_{\mathfrak{p}}\left(l_{2}(x)\right)$ are both odd. We have to show in this situation that $a l_{3}(x) \in k_{\nu_{\mathrm{p}}}^{*}$ is a square.

For this, as $\nu_{\mathfrak{p}}\left(l_{1}(x)\right)$ is odd and $\nu_{\mathfrak{p}}\left(b v^{2}(x)\right)$ is even, the second equation implies

$$
\begin{aligned}
\nu_{\mathfrak{p}}\left(l_{3}(x) l_{4}(x)\right) & =\min \left(\nu_{\mathfrak{p}}\left(b v^{2}(x)\right), \nu_{\mathfrak{p}}\left(l_{1}(x)\right)\right) \\
& \leq \nu_{\mathfrak{p}}\left(l_{1}(x)\right),
\end{aligned}
$$

hence

$$
\nu_{\mathfrak{p}}\left(l_{3}(x)\right) \leq \nu_{\mathfrak{p}}\left(l_{3}(x) l_{4}(x)\right) \leq \nu_{\mathfrak{p}}\left(l_{1}(x)\right)<\nu_{\mathfrak{p}}\left(l_{1}(x) l_{2}(x)\right) .
$$

Note here that $\nu_{\mathfrak{p}}\left(l_{2}(x)\right) \geq 1$, the valuation of $l_{2}(x)$ being odd. Consequently, the first equation implies that $a l_{3}(x)$ is a square in $k_{\nu_{\mathrm{p}}}^{*}$. This completes the proof.

\section{EXAMPLES}

Recall [DW, Definition 2.1] (cf. [PR, §7.1]) that strong approximation off a certain set $S$ of places is said to hold for an algebraic variety $U$ defined over a number field $k$ if the image of the set $U(k)$ of $k$-rational points on $U$ is dense in the space $U\left(\mathbb{A}_{k}^{S}\right)$ of adelic points on $U$ outside $S$.

Del Pezzo surfaces of degree four.

In our first example, only a transcendental Brauer class occurs.

Example 6.1. Let $X \subset \mathbf{P}_{\mathbb{Q}}^{4}$ be given by the system of equations

$$
\begin{aligned}
X_{0} X_{1}+X_{2}^{2} & =X_{4} X_{3}, \\
X_{3}\left(2 X_{1}+X_{2}+X_{3}\right)+X_{0}^{2} & =X_{4} X_{1}
\end{aligned}
$$

and put $U:=X \backslash H$, for the hyperplane $H:=V\left(X_{4}\right) \subset \mathbf{P}_{\mathbb{Q}}^{4}$.

i) Then $X$ is a del Pezzo surface of degree four. 
ii) $D:=H \cap X$ is a non-singular curve of genus one. Furthermore,

$$
D(\mathbb{Q})=\{(0: 1: 0: 0: 0),(0:-1: 0: 2: 0)\} .
$$

iii) The manifold $X(\mathbb{R})$ is connected, its submanifold $U(\mathbb{R})$ is connected, too, and $U$ is strongly unobstructed at $\infty$.

iv) One has $\operatorname{Br}(U) / \operatorname{Br}_{0}(U) \cong \mathbb{Z} / 2 \mathbb{Z}$, a representative of the nontrivial element being

$$
\tau:\left(\frac{X_{1}}{X_{4}}, \frac{X_{3}}{X_{4}} ;-1\right) \text {. }
$$

Moreover, $\operatorname{Br}_{1}(U) / \operatorname{Br}_{0}(U)=0$. I.e., the Brauer class $\tau$ is transcendental.

Proof. i) and ii) magma reports that both $X$ and $D$ are non-singular. More precisely, $D$ is isomorphic to the elliptic curve $E: y^{2} z+x y z=x^{3}+4 x z^{2}$, the Mordell-Weil group of which is isomorphic to $\mathbb{Z} / 2 \mathbb{Z}$.

iii) The pencil of quadrics in $\mathbf{P}^{4}$ corresponding to $X$ contains only one degenerate quadric that is defined over $\mathbb{R}$. According to [VAV, Theorem 3.4 and Lemma 5.1], this implies $\operatorname{Br}\left(X_{\mathbb{R}}\right) / \operatorname{Br}(\mathbb{R})=0$, which is enough to show that $X(\mathbb{R})$ is connected [Si, Théorème 1.4].

As $X$ has no real point such that $x_{1}=x_{4}=0$, the rational map $\pi: X-\rightarrow \mathbf{P}^{1}$, given by $x \mapsto x_{1} / x_{4}$, is well-defined on real points. Furthermore, $U(\mathbb{R})=\pi^{-1}\left(\mathbf{A}^{1}(\mathbb{R})\right)$. A calculation using Gröbner bases shows that $\pi$ has exactly 10 singular fibres, among which only two, those over 0 and $\infty$, are real. Finally, experiments with $t=1$ and $t=-1$ indicate that the fibres $\pi^{-1}(t)$, for $t>0$ as well as for $t<0$, are genus-one curves with a connected set of real points. As connectedness of $\pi^{-1}(0)$ is directly checked, this is enough to guarantee that $U(\mathbb{R})$ is connected.

Since $U(\mathbb{R})$ is connected and $D$ is a non-singular, geometrically irreducible scheme having real points, Theorem 2.7 shows that $U$ is strongly unobstructed at the real place.

iv) A calculation using Gröbner bases shows that the Galois group operating on the 16 lines on $X$ is the full $W\left(D_{5}\right)$ of order 1920. Therefore, Theorem 4.8.b.i) immediately implies that $\operatorname{Br}_{1}(U) / \operatorname{Br}_{0}(U)=0$.

On the other hand, we have $\# D(\mathbb{Q})=2$. Moreover, $E$ has two $\mathbb{Q}$-rational 4 -isogenies, but no nontrivial $n$-isogenies for any other $n \geq 3$. As the isogenous curves have no proper $\mathbb{Q}$-rational 4-torsion points, Corollary 4.10 shows that there is an injection $\operatorname{Br}(U) / \operatorname{Br}_{1}(U) \hookrightarrow \mathbb{Z} / 2 \mathbb{Z}$. Consequently, $\operatorname{Br}(U) / \operatorname{Br}_{0}(U)$ is of order at most 2. As far as a concrete description is asked for, Theorem 5.5 applies. It shows that $\left(\frac{X_{1}}{X_{4}}, \frac{X_{3}}{X_{4}} ;-1\right)$ defines a Brauer class on $U$.

In order to prove that this is indeed a nontrivial class, the simplest argument is probably that its local evaluation map $\mathrm{ev}_{\tau, \nu_{2}}$ is non-constant, which we show in Example 6.2.i) below.

Example 6.2 (continued). Let $X, U$, and $\tau$ be as above. Moreover, let $\mathscr{X} \subset \mathbf{P}_{\mathbb{Z}}^{4}$ be defined by the same system of equations as $X$ and put $\mathscr{U}:=\mathscr{X} \backslash \mathscr{H}$, for $\mathscr{H}:=V\left(X_{4}\right) \subset \mathbf{P}_{\mathbb{Z}}^{4}$. Then 
i) For all primes $p \neq 2$ including the archimedean one, the local evaluation map $\mathrm{ev}_{\tau, \nu_{p}}: \mathscr{U}\left(\mathbb{Z}_{p}\right) \rightarrow \mathbb{Q} / \mathbb{Z}$ is constantly zero. On the other hand, ev $\operatorname{ev}_{\tau, \nu_{2}}: \mathscr{U}\left(\mathbb{Z}_{2}\right) \rightarrow \mathbb{Q} / \mathbb{Z}$ is non-constant.

ii) For $p=17$, even the local evaluation map $\operatorname{ev}_{\tau, \nu_{17}}: U\left(\mathbb{Q}_{17}\right) \rightarrow \mathbb{Q} / \mathbb{Z}$ is constantly zero.

Proof. i) For all primes with the exception of the infinite one, this follows directly from Lemma 5.9. To prove constancy for $\mathrm{ev}_{\tau, \nu_{\infty}}$, we have to show that, for a real point $x=\left(x_{0}: \ldots: x_{3}: 1\right) \in U(\mathbb{R})$, the coordinates $x_{1}$ and $x_{3}$ cannot both be negative.

Assume the contrary. In that case, the first equation shows $x_{0}>0$. Moreover, $x_{2}^{2}+\left|x_{3}\right|=\left|x_{0} x_{1}\right|$ and hence $x_{2}^{2} \leq\left|x_{0} x_{1}\right|$, implying $x_{2} \leq x_{0}$ or $x_{2} \leq-x_{1}$. In the first case,

$$
\begin{aligned}
x_{3}\left(2 x_{1}+x_{2}+x_{3}\right)+x_{0}^{2} & =2 x_{1} x_{3}+x_{2} x_{3}+x_{3}^{2}+x_{0}^{2} \\
& \geq 2 x_{1} x_{3}+x_{0} x_{3}+x_{3}^{2}+x_{0}^{2}>2 x_{1} x_{3}+\left(x_{0}+x_{3}\right)^{2}>0,
\end{aligned}
$$

in contradiction to the second equation. And in the second case, the same is true, since $x_{2} x_{3} \geq-x_{1} x_{3}$.

Finally, non-constancy of $\mathrm{ev}_{\tau, \nu_{2}}$ may easily be seen directly. Indeed, for the integral point $x=(-1:-1:-1: 2: 1) \in \mathscr{U}\left(\mathbb{Z}_{2}\right)$, one has $\left(x_{1}, x_{3}\right)_{2}=(-1,2)_{2}=1$, hence $\mathrm{ev}_{\tau, \nu_{2}}(x)=0$. On the other hand, the $\mathbb{Z}_{2}$-valued point $x^{\prime}=\left(\frac{2}{5}: \frac{2}{5}: \frac{1}{5}: \frac{1}{5}: 1\right) \in \mathscr{U}\left(\mathbb{Z}_{2}\right)$ yields $\left(x_{1}, x_{3}\right)_{2}=\left(\frac{2}{5}, \frac{1}{5}\right)_{2}=-1$ and $\mathrm{ev}_{\tau, \nu_{2}}(x)=\frac{1}{2}$.

ii) The local evaluation map $\mathrm{ev}_{\tau, \nu_{17}}$ is locally constant with respect to the 17-adic topology on $U\left(\mathbb{Q}_{17}\right)$. Thus, it suffices to prove the claim for points $x=\left(x_{0}: \ldots: x_{4}\right) \in U\left(\mathbb{Q}_{17}\right)$ such that $x_{1} \neq 0$ and $x_{3} \neq 0$. Moreover, the assumption implies that $x_{4} \neq 0$. Let us assume that the coordinates are normalised such that $x_{0}, \ldots, x_{4} \in \mathbb{Z}_{17}$ and at least one is a unit.

If $x_{4}$ is a unit then $x$ defines a $\mathbb{Z}_{17}$-valued point on $\mathscr{U}$. In this case, $\operatorname{ev}_{\tau, \nu_{17}}(x)=0$, as follows from i). Thus, assume that $17 \mid x_{4}$. Then $x$ reduces modulo 17 to a point on the reduction $D_{17}$ of the curve $D$. A direct inspection shows that $D_{17}$ has exactly 17 $\mathbb{F}_{17}$-rational points, that $\xi_{1} \xi_{3} \neq 0$ for exactly 14 of these, and that $\xi_{1} / \xi_{3} \in \mathbb{F}_{17}^{*}$ is a square for each of them. Therefore, if $17 \nmid x_{1}$ and $17 \nmid x_{3}$ then $x_{1} / x_{3} \in \mathbb{Q}_{17}^{*}$ is a square and, consequently, $\left(\frac{x_{1}}{x_{4}}, \frac{x_{3}}{x_{4}}\right)_{17}=\left(-\frac{x_{1}}{x_{3}}, \frac{x_{3}}{x_{4}}\right)_{17}=1$.

Moreover, if $17 \mid x_{4}$ and $17 \mid x_{3}$ then the equations of the surface imply $17 \mid x_{0}$ and $17 \mid x_{2}$, so that $x_{1}$ is a unit. As the second equation shows that $-x_{3}\left(2 x_{1}+x_{2}+x_{3}\right)$ is a norm from $\mathbb{Q}_{17}\left(\sqrt{x_{1} / x_{4}}\right)$, one has $\left(\frac{x_{1}}{x_{4}}, \frac{x_{3}}{x_{4}}\right)_{17}=\left(\frac{x_{1}}{x_{4}}, \frac{-2 x_{1}-x_{2}-x_{3}}{x_{4}}\right)_{17}=\left(\frac{x_{1}}{x_{4}}, \frac{-2 x_{1}}{x_{4}}\right)_{17}=1$.

Finally, assume that $17 \mid x_{4}$ and $17 \mid x_{1}$. Then the equations of the surface imply $17 \mid x_{2}$ and that $x_{0}$ and $x_{3}$ are units such that $x_{0}^{2}+x_{3}^{2} \equiv 0(\bmod 17)$. This is enough to ensure that $x_{0} / x_{3} \in \mathbb{Q}_{17}^{*}$ is a square. Since the first equation shows that $-x_{0} x_{1}$ is a norm from $\mathbb{Q}_{17}\left(\sqrt{x_{3} / x_{4}}\right)$, one has $\left(\frac{x_{1}}{x_{4}}, \frac{x_{3}}{x_{4}}\right)_{17}=\left(-\frac{x_{0}}{x_{4}}, \frac{x_{3}}{x_{4}}\right)_{17}=\left(-\frac{x_{3}}{x_{4}}, \frac{x_{3}}{x_{4}}\right)_{17}=1$, which completes the proof. 
Remarks 6.3. i) Thus, the Brauer class $\tau$ causes a violation of strong approximation on $X$ off $\{17, \infty\}$. In fact, a $\mathbb{Z}\left[\frac{1}{17}\right]$-valued point such that $x_{1} \neq 0$ and $x_{3} \neq 0$ must necessarily fulfil $\left(\frac{x_{1}}{x_{4}}, \frac{x_{3}}{x_{4}}\right)_{2}=1$, although not all $\mathbb{Z}_{2}$-valued points satisfy this relation.

ii) This restriction applies, in particular, to integral points.

iii) There are infinitely many integral points on $\mathscr{U}$. Indeed, the curve defined by $V\left(X_{3}\right)$ yields the family

$$
\left(-n^{2}: n^{4}: \pm n^{3}: 0: 1\right)
$$

On the other hand, the curve defined by $V\left(2 X_{1}+X_{2}+X_{3}\right)$ is elliptic. It carries the six integral points $(0: 0: 0: 0: 1),(0: 0:-1: 1: 1),(-2: 4:-1:-7: 1),(-2: 4: 0:-8: 1)$, $(-14: 196:-49:-343: 1),(-14: 196: 48:-440: 1)$, and no others.

iv) A rather naively implemented search for integral points on $\mathscr{U}$ delivered 28 of height $<50000$ that are not of the forms mentioned above. These are the following, $(0:-2: 1: 1: 1),(-1:-1:-1: 2: 1),(-1:-3: 1: 4: 1),(4:-8: 6: 4: 1),(-6: 4: 4:-8: 1)$, $(-8: 18:-11:-23: 1),(14:-28: 20: 8: 1),(16:-56: 30: 4: 1),(76:-696:-230: 4: 1)$, $(-97: 521:-223:-808: 1),(-105: 1413: 381:-3204: 1),(263:-829: 467: 62: 1)$, $(-556: 912: 712:-128: 1),(-708: 1278:-951:-423: 1),(839:-1595: 1157: 444: 1)$, $(1004:-1648:-1288: 4352: 1),(-2073: 3573:-2721:-2988: 1),(2238:-6876: 3924: 9288: 1)$, $(-2264: 3840:-2948:-3056: 1),(-2916: 5832: 4122:-15228: 1),(3324:-15678:-7219: 289: 1)$, $(3879:-6183:-4899: 16344: 1),(-5450: 14688: 8947:-791: 1),(-5809: 11231:-8077:-2950: 1)$, $(8908:-16476: 12115: 5017: 1),(-10194: 6948: 8415:-15687: 1),(22238:-38044: 29087: 31097: 1)$, and $(-26396: 44152:-34138:-33148: 1)$.

v) The exceptional behaviour at the prime 17 is possible only because $D$ has bad reduction at the prime 17 . The curve $D_{17}$ has exactly one singular point, at $(1: 1: 13: 1: 0)$. Using Gröbner bases, one may easily verify that, on $D_{17}$, there is the relation

$$
\frac{X_{1}}{X_{3}}=8\left(\frac{X_{0} X_{2} X_{3}-2 X_{2}^{3}+X_{0} X_{2}^{2}+X_{0}^{2} X_{2}}{X_{0}^{2}\left(4 X_{0}+X_{2}\right)}\right)^{2} .
$$

Example 6.4. Let $\mathscr{X}^{\prime} \subset \mathbf{P}_{\mathbb{Z}}^{4}$ be given by the system of equations

$$
\begin{aligned}
X_{0}\left(8 X_{1}+3 X_{4}\right)+X_{2}^{2} & =X_{4}\left(8 X_{3}+2 X_{4}\right), \\
\left(8 X_{3}+2 X_{4}\right)\left(16 X_{1}+X_{2}+8 X_{3}+8 X_{4}\right)+X_{0}^{2} & =X_{4}\left(8 X_{1}+3 X_{4}\right) .
\end{aligned}
$$

Put $\mathscr{U}^{\prime}:=\mathscr{X}^{\prime} \backslash \mathscr{H}$ for the hyperplane $\mathscr{H}:=V\left(X_{4}\right) \subset \mathbf{P}_{\mathbb{Z}}^{4}$ and denote the generic fibre of $\mathscr{U}^{\prime}$ by $U^{\prime}$.

Then $\mathscr{U}^{\prime}\left(\mathbb{Z}_{p}\right) \neq \emptyset$ for every prime number $p$ and $U^{\prime}(\mathbb{Q}) \neq \emptyset$, but there are no $\mathbb{Z}\left[\frac{1}{17}\right]$-valued points, $\mathscr{U}^{\prime}\left(\mathbb{Z}\left[\frac{1}{17}\right]\right)=\emptyset$. I.e, the Hasse principle for $\mathbb{Z}\left[\frac{1}{17}\right]$-valued points is violated. In particular, a failure of the integral Hasse principle occurs. The violations can be explained by a transcendental Brauer class.

Proof. There is a morphism $\pi: \mathscr{U}^{\prime} \rightarrow \mathscr{U}$ to the scheme from Example 6.2 that is given by

$$
\pi:\left(x_{0}: x_{1}: x_{2}: x_{3}: 1\right) \mapsto\left(x_{0}:\left(8 x_{1}+3\right): x_{2}:\left(8 x_{3}+2\right): 1\right)
$$


This morphism yields bijections between the sets of $\mathbb{Q}$-rational points, as well as between the sets of $\mathbb{Z}_{p}$-valued points for $p \neq 2$. In particular, $U^{\prime}(\mathbb{Q}) \neq \emptyset$ and $\mathscr{U}^{\prime}\left(\mathbb{Z}_{p}\right) \neq \emptyset$, except possibly for $p \neq 2$. Moreover, there is the $\mathbb{Z}_{2}$-valued point $\left(3:-\frac{4}{3}: 5: 0: 1\right) \in \mathscr{U}^{\prime}\left(\mathbb{Z}_{2}\right)$.

There is, however, no $\mathbb{Z}\left[\frac{1}{17}\right]$-valued point on $\mathscr{U}^{\prime}$. In fact, the image of such a point would fulfil $\left(8 x_{1}+3,8 x_{3}+2\right)_{2}=(3,2)_{2}=-1$, in contradiction to what was proven about $\mathscr{U}$, cf. Remark 6.3.i).

Remarks 6.5. i) Observe that the non-existence of integral points on $\mathscr{U}^{\prime}$ may not be explained by a lack of real points. In fact, the $\mathbb{Q}$-scheme $U^{\prime}$ is strongly unobstructed at $\infty$, simply because $U$ is.

ii) The result may be formulated in a more classical manner as follows. The pair $\left(q_{1}, q_{2}\right)$ of quadratic polynomials in four variables, consisting of

$$
\begin{aligned}
& q_{1}:=\quad X_{0}\left(8 X_{1}+3\right)+X_{2}^{2}-\left(8 X_{3}+2\right), \\
& q_{2}:=\left(8 X_{3}+2\right)\left(16 X_{1}+X_{2}+8 X_{3}+8\right)+X_{0}^{2}-\left(8 X_{1}+3\right) \text {, }
\end{aligned}
$$

does not represent $(0,0)$ integrally, although it does so over the rationals and in $p$-adic integers for every prime number $p$.

Blowing up a point. A cubic surface.

Example 6.6. Let $\mathscr{S} \subset \mathbf{P}_{\mathbb{Z}}^{3}$ be given by the equation

$$
Y_{0}^{3}+Y_{0} Y_{1} Y_{2}+Y_{0} Y_{2}^{2}-2 Y_{1}^{2} Y_{2}+Y_{1}^{2} Y_{3}+2 Y_{2}^{2} Y_{3}-Y_{2} Y_{3}^{2}=0
$$

and put $\mathscr{V}:=\mathscr{S} \backslash \mathscr{E}$, for the hyperplane $\mathscr{E}:=V\left(Y_{3}\right) \subset \mathbf{P}_{\mathbb{Z}}^{3}$.

Then every integral point $\left(y_{0}: y_{1}: y_{2}: 1\right) \in \mathscr{V}(\mathbb{Z})$ such that $y_{0} y_{2} \neq 0$ satisfies the condition that $\left(y_{0}\left(y_{2}-y_{1}^{2}\right), y_{2}\right)_{2}=1$ or $\operatorname{gcd}\left(y_{0}, 2 y_{2}-1\right)>1$.

Proof. The scheme $\mathscr{S}$ is obtained from the scheme $\mathscr{X}$ of Example 6.1 by blowing up the point $(0: 1: 0: 0: 0)$. From the computational viewpoint, this means to eliminate $X_{1}$ from the equations defining $\mathscr{X}$. Moreover, we replaced the coordinate sections $X_{0}, X_{2}, X_{3}, X_{4}$, in this order, by $Y_{0}, Y_{1}, Y_{2}$, and $Y_{3}$. An integral point on $\mathscr{V}$ thus corresponds to a $\mathbb{Q}$-rational point $\left(x_{0}: x_{1}: x_{2}: x_{3}: 1\right) \in U(\mathbb{Q})$ such that $x_{0}, x_{2}$, and $x_{3}$ are integers, but $x_{1}$ not necessarily.

If, however, $\operatorname{gcd}\left(x_{0}, 2 x_{3}-1\right)=1$ then the equations $x_{0} \cdot x_{1}=x_{3}-x_{2}^{2}$ and $\left(2 x_{3}-1\right) \cdot x_{1}=-\left(x_{0}^{2}+x_{2} x_{3}+x_{3}^{2}\right)$ together imply that $x_{1}$ has to be an integer, as well. Then, by Example 6.2.i), $\left(x_{1}, x_{3}\right)_{2}=1$. The assertion follows, as $\mathscr{S}$ does not have integral points such that $y_{2}-y_{1}^{2}=0$, but $y_{0} y_{2} \neq 0$.

Remarks 6.7. i) Write $S \subset \mathbf{P}_{\mathbb{Q}}^{3}$ and $E \subset \mathbf{P}_{\mathbb{Q}}^{3}$ for the generic fibres of $\mathscr{S}$ and $\mathscr{E}$, respectively, and put $V:=S \backslash E$. Then, under the blow-down morphism $\pi: S \rightarrow X$, one has $\pi^{-1}(H)=E \cup L$ and, hence, $\pi^{-1}(U)=V \backslash L$, for $L \subset S$ the line given by $Y_{0}=2 Y_{2}-Y_{3}=0$. Note that a point on the hyperplane $H$ has been blown up.

The effect deduced above by an elementary argument is, of course, that of the Brauer class $\pi^{*} \tau \in \operatorname{Br}(V \backslash L)$. The points such that $\operatorname{gcd}\left(y_{0}, 2 y_{2}-1\right)=1$ are exactly those that are integral with respect to the obvious integral model of $V \backslash L$. 
ii) Blowing up a point outside $H$ leads to $\pi^{-1}(H)$ being a non-plane genus-one curve on $S$. In this case, the corresponding notion of integrality for points is further away from the classical meaning.

iii) There exist integral points on $\mathscr{V}$ of all three kinds that are allowed by the statements above. For instance, for $(-1:-1: 2: 1)$, the gcd is 1 and the Hilbert symbol is 1 . On the other hand, for $(-17: 15:-8: 1)$, the gcd is $17>1$ and the Hilbert symbol is 1 , while for $(3: 5: 2: 1)$, the gcd is $3>1$ and the Hilbert symbol is equal to $(-1)$.

Example 6.8. Let $\mathscr{S}^{\prime} \subset \mathbf{P}_{\mathbb{Z}}^{3}$ be given by the equation

$$
\begin{aligned}
128 Y_{0}^{3}+144 Y_{0}^{2} Y_{3}+32 Y_{0} Y_{1} Y_{2}+8 Y_{0} Y_{1} Y_{3}+128 Y_{0} Y_{2}^{2}+80 Y_{0} Y_{2} Y_{3}+66 Y_{0} Y_{3}^{2} \\
-16 Y_{1}^{2} Y_{2}-3 Y_{1}^{2} Y_{3}-4 Y_{1} Y_{2} Y_{3}+80 Y_{2}^{2} Y_{3}+40 Y_{2} Y_{3}^{2}+12 Y_{3}^{3}=0
\end{aligned}
$$

and put $\mathscr{V}^{\prime}:=\mathscr{S}^{\prime} \backslash \mathscr{E}$, for the hyperplane $\mathscr{E}:=V\left(Y_{3}\right) \subset \mathbf{P}_{\mathbb{Z}}^{3}$.

Then every integral point $\left(y_{0}: y_{1}: y_{2}: 1\right) \in \mathscr{V}^{\prime}(\mathbb{Z})$ satisfies $\operatorname{gcd}\left(8 y_{0}+3,16 y_{2}+3\right)>1$.

Proof. There is a morphism $\pi: \mathscr{V}^{\prime} \rightarrow \mathscr{V}$ to the scheme from Example 6.6 that is given by

$$
\pi:\left(y_{0}: y_{1}: y_{2}: 1\right) \mapsto\left(\left(8 y_{0}+3\right):\left(2 y_{1}+1\right):\left(8 y_{2}+2\right): 1\right) .
$$

As $\left(8 y_{0}+3\right)\left[\left(8 y_{2}+2\right)-\left(2 y_{1}+1\right)^{2}\right] \equiv 3(\bmod 8)$ and $8 y_{2}+2 \equiv 2(\bmod 8)$, the Hilbert symbol is always equal to $(-1)$, as required.

Remark 6.9. The surface $\mathscr{V}^{\prime}$ contains infinitely many integral points. Indeed, define the two sequences $c$ and $c^{\prime}$ in $\mathbb{Z}^{3}$ recursively by

$$
\begin{aligned}
& c_{1}:=[0,-2,0], \quad c_{2}:=[-48,170,-24], \quad c_{i+2}:=-110 c_{i+1}-c_{i}-[48,48,24], \\
& c_{1}^{\prime}:=[0,2,0], \quad c_{2}^{\prime}:=[-48,-266,-24], \quad c_{i+2}^{\prime}:=-110 c_{i+1}^{\prime}-c_{i}^{\prime}-[48,48,24] .
\end{aligned}
$$

Then, for each $i \in \mathbb{N},\left(c_{i 1}: c_{i 2}: c_{i 3}: 1\right) \in \mathscr{V}^{\prime}(\mathbb{Z})$ and $\left(c_{i 1}^{\prime}: c_{i 2}^{\prime}: c_{i 3}^{\prime}: 1\right) \in \mathscr{V}^{\prime}(\mathbb{Z})$. Observe that the intersection of $\mathscr{S}^{\prime}$ with the plane given by $Y_{0}=2 Y_{2}$ contains the exceptional line and, therefore, splits off a conic.

There are further integral points on $\mathscr{V}^{\prime}$ not being of this particular form, for instance $(-1536: 5414:-803: 1)$ and $(20706:-344632: 534: 1)$. Moreover, both are the smallest members of infinite sequences of integral points of the same kind as (4). However, the second member of the sequence starting at $(-1536: 5414:-803: 1)$ involves 1340-digit integers, already.

\section{MORE EXAMPLES}

A del Pezzo surface of degree four.

In the example below, an algebraic Brauer class interacts with transcendental ones.

Example 7.1. Let $X \subset \mathbf{P}_{\mathbb{Q}}^{4}$ be given by the system of equations

$$
\begin{aligned}
X_{0} X_{1}+X_{2}^{2} & =X_{4} X_{3}, \\
X_{3}\left(X_{1}+X_{3}\right)+X_{0}^{2} & =X_{4} X_{1}
\end{aligned}
$$


and put $U:=X \backslash H$, for the hyperplane $H:=V\left(X_{4}\right) \subset \mathbf{P}_{\mathbb{Q}}^{4}$.

i) Then $X$ is a del Pezzo surface of degree four.

ii) $D:=H \cap X$ is a non-singular curve of genus one. Furthermore,

$$
D(\mathbb{Q})=\{(0: 1: 0: 0: 0),(0: 1: 0:-1: 0)\} .
$$

iii) The manifold $X(\mathbb{R})$ is connected, its submanifold $U(\mathbb{R})$ consists of two connected components, and $U$ is strongly unobstructed at $\infty$.

iv) One has $\operatorname{Br}(U) / \operatorname{Br}_{0}(U) \cong(\mathbb{Z} / 2 \mathbb{Z})^{2}$, the three nontrivial elements being represented by

$$
\begin{aligned}
\alpha: & \left(\frac{X_{1}}{X_{4}},-1 ;-1\right), \\
\tau: & \left(\frac{X_{1}}{X_{4}}, \frac{X_{3}}{X_{4}} ;-1\right), \quad \text { and } \\
\alpha+\tau: & \left(\frac{X_{1}}{X_{4}},-\frac{X_{3}}{X_{4}} ;-1\right) .
\end{aligned}
$$

Moreover, $\operatorname{Br}_{1}(U) / \operatorname{Br}_{0}(U)=\langle\alpha\rangle \cong \mathbb{Z} / 2 \mathbb{Z}$.

Proof. i) and ii) magma reports that both $X$ and $D$ are non-singular. More precisely, $D$ is isomorphic to the elliptic curve $E: y^{2} z=x^{3}+x z^{2}$ of $j$-invariant 1728 , an isomorphism $\iota: D \rightarrow E$ being given by

$$
\left(X_{0}: X_{1}: X_{2}: X_{3}: 0\right) \mapsto\left(X_{2}^{2}:-X_{1} X_{2}:-X_{1} X_{3}\right)
$$

This explains, in particular, why $D$ has no $\mathbb{Q}$-rational points other than the two obvious ones.

iii) The pencil of quadrics in $\mathbf{P}^{4}$ corresponding to $X$ contains exactly three degenerate quadrics that are defined over $\mathbb{R}$. Among the corresponding rank-four discriminants, two are negative but the third one is positive. According to [VAV, Theorem 3.4 and Lemma 5.1], this already implies that $\operatorname{Br}\left(X_{\mathbb{R}}\right) / \operatorname{Br}(\mathbb{R})=0$. Consequently, $X(\mathbb{R})$ is connected [ $\mathrm{Si}$, Théorème 1.4].

As the cubic polynomial $x^{3}+x$ has only one real root, we see that $D(\mathbb{R})$ is connected, too. Such a curve may cut $X(\mathbb{R})$ into not more than two components. On the other hand, $U(\mathbb{R})$ is clearly disconnected. Indeed, for a real point $\left(x_{0}: x_{1}: x_{2}: x_{3}: 1\right) \in U(\mathbb{R})$, the second equation implies $\left(x_{3}+\frac{x_{1}}{2}\right)^{2}+x_{0}^{2}=\frac{1}{4}\left[x_{1}^{2}+4 x_{1}\right]$, hence $x_{1} \geq 0$ or $x_{1} \leq-4$. And there exist points of both kinds, for example $(-1: 1: 0:-1: 1)$ and $(0:-4: \sqrt{2}: 2: 1)$.

Since $D$ is a non-singular, geometrically irreducible curve having real points and both components of $U(\mathbb{R})$ have limit points in $D(\mathbb{R})$, Theorem 2.7 yields that $U$ is strongly unobstructed at the real place.

iv) A calculation using Gröbner bases shows that the Galois group operating on the 16 lines on $X$ is of order exactly 384. Thus, Theorem 4.8.b.ii) immediately implies that $\operatorname{Br}_{1}(U) / \operatorname{Br}_{0}(U) \cong \mathbb{Z} / 2 \mathbb{Z}$. Moreover, the second equation is only of rank 4 and may be written in the form $X_{1}\left(X_{4}-X_{3}\right)-X_{3}^{2}-X_{0}^{2}=0$. Therefore, Theorem 5.4 shows that $\left(\frac{X_{1}}{X_{4}},-1 ;-1\right)$ defines a nontrivial algebraic Brauer class on $U$. 
On the other hand, we have $\# D(\mathbb{Q})=2$. Again, $E$ has two nontrivial 4-isogenies, but no nontrivial $n$-isogenies for any other $n \geq 3$. Only one of the isogenous curves has a $\mathbb{Q}$-rational 4-torsion point, which, however, is not in the image of the 4-torsion of $E$. Hence, Corollary 4.10 provides us with an injection $\operatorname{Br}(U) / \operatorname{Br}_{1}(U) \hookrightarrow \mathbb{Z} / 2 \mathbb{Z}$. Consequently, $\operatorname{Br}(U) / \operatorname{Br}_{0}(U)$ is of order at most 4. As far as a concrete description is asked for, Theorem 5.5 applies. It shows that $\left(\frac{X_{1}}{X_{4}}, \frac{X_{3}}{X_{4}} ;-1\right)$ defines a Brauer class on $U$.

In order to prove that this is indeed a transcendental class, we need to show that $\frac{X_{1}}{X_{3}}$ is not the square of a rational function on $D_{\overline{\mathbb{Q}}}$. For this, we may argue as follows. One has

$$
\begin{aligned}
-\frac{X_{1}}{X_{3}} & =-\frac{X_{1} X_{3}}{X_{3}^{2}} \\
& =\frac{X_{0}^{2}+X_{3}^{2}}{X_{3}^{2}} \\
& =\frac{X_{0}^{2} X_{1}^{2}+X_{1}^{2} X_{3}^{2}}{X_{1}^{2} X_{3}^{2}} \\
& =\frac{X_{2}^{4}+X_{1}^{2} X_{3}^{2}}{X_{1}^{2} X_{3}^{2}}
\end{aligned}
$$

and this is the product of $F:=\frac{X_{2}^{2}+i X_{1} X_{3}}{X_{1} X_{3}}$ together with its complex conjugate. One directly finds that

$$
\operatorname{div} F=2[(1: 0: 0: i: 1)]-2[(0: 1: 0: 0: 1)],
$$

hence

$$
\operatorname{div} \bar{F}=2[(1: 0: 0:-i: 1)]-2[(0: 1: 0: 0: 1)]
$$

and

$$
\operatorname{div}\left(\frac{X_{1}}{X_{3}}\right)=2[(1: 0: 0: i: 1)]+2[(1: 0: 0:-i: 1)]-4[(0: 1: 0: 0: 1)] .
$$

In particular, $(1: 0: 0: i: 1)-(0: 1: 0: 0: 1) \in J(D)(\overline{\mathbb{Q}})$ is a proper 2-torsion point, and $(1: 0: 0:-i: 1)-(0: 1: 0: 0: 1) \in J(D)(\overline{\mathbb{Q}})$ is another.

Therefore, their sum $(1: 0: 0: i: 1)+(1: 0: 0:-i: 1)-2(0: 1: 0: 0: 1) \in J(D)(\overline{\mathbb{Q}})$ is the third 2-torsion point, in particular it is non-zero. As a consequence of this, we see that $\frac{X_{1}}{X_{3}}$ can not be the square of a rational function on $D_{\overline{\mathbb{Q}}}$, as required.

Example 7.2 (continued). Let $X, U, \alpha$, and $\tau$ be as above. Moreover, let $\mathscr{X} \subset \mathbf{P}_{\mathbb{Z}}^{4}$ be defined by the same system of equations as $X$ and put $\mathscr{U}:=\mathscr{X} \backslash \mathscr{H}$, for $\mathscr{H}:=V\left(X_{4}\right) \subset \mathbf{P}_{\mathbb{Z}}^{4}$. Then

i) for all primes $p \neq 2, \infty$, the local evaluation maps $\operatorname{ev}_{\alpha, \nu_{p}}: \mathscr{U}\left(\mathbb{Z}_{p}\right) \rightarrow \mathbb{Q} / \mathbb{Z}$, $\mathrm{ev}_{\tau, \nu_{p}}: \mathscr{U}\left(\mathbb{Z}_{p}\right) \rightarrow \mathbb{Q} / \mathbb{Z}$, and $\mathrm{ev}_{\alpha+\tau, \nu_{p}}: \mathscr{U}\left(\mathbb{Z}_{p}\right) \rightarrow \mathbb{Q} / \mathbb{Z}$ are constantly zero.

ii) The local evaluation map $\operatorname{ev}_{\tau, \nu_{\infty}}: U(\mathbb{R}) \rightarrow \frac{1}{2} \mathbb{Z} / \mathbb{Z}$ is constantly zero, but $\mathrm{ev}_{\tau, \nu_{2}}: \mathscr{U}\left(\mathbb{Z}_{2}\right) \rightarrow \mathbb{Q} / \mathbb{Z}$ is non-constant.

iii) The local evaluation map $\operatorname{ev}_{\alpha+\tau, \nu_{\infty}}: U(\mathbb{R}) \rightarrow \frac{1}{2} \mathbb{Z} / \mathbb{Z}$ is non-constant, but $\mathrm{ev}_{\alpha+\tau, \nu_{2}}: \mathscr{U}\left(\mathbb{Z}_{2}\right) \rightarrow \mathbb{Q} / \mathbb{Z}$ is constantly zero.

iv) The maps $\operatorname{ev}_{\alpha, \nu_{2}}: \mathscr{U}\left(\mathbb{Z}_{2}\right) \rightarrow \mathbb{Q} / \mathbb{Z}$ and $\mathrm{ev}_{\alpha, \nu_{\infty}}: U(\mathbb{R}) \rightarrow \frac{1}{2} \mathbb{Z} / \mathbb{Z}$ are both nonconstant. 
v) For $p \equiv 1(\bmod 4)$, even the local evaluation map $\operatorname{ev}_{\alpha, \nu_{p}}: U\left(\mathbb{Q}_{p}\right) \rightarrow \mathbb{Q} / \mathbb{Z}$ is constantly zero.

Proof. i) For $\mathrm{ev}_{\tau, \nu_{p}}$, this is directly Lemma 5.9. And for $\mathrm{ev}_{\alpha, \nu_{p}}$, Lemma 5.7 applies, since $d=-1$, the linear forms $X_{1}, X_{4}-X_{3}, X_{3}$, and $X_{0}$ are linearly independent modulo every prime $p$, and the cusp $(0: 0: 1: 0: 0)$ does not satisfy the second equation $X_{0} X_{1}+X_{2}^{2}=X_{4} X_{3}$ modulo any prime.

ii) The second equation excludes the possibility that $X_{1}$ and $X_{3}$ might both be negative. Hence, $\mathrm{ev}_{\tau, \nu_{\infty}}$ is constantly zero.

Furthermore, for the integral point $x=(-1: 1: 0:-1: 1) \in \mathscr{U}\left(\mathbb{Z}_{2}\right)$, one has $\left(x_{1}, x_{3}\right)_{2}=(1,-1)_{2}=1$ and thus $\operatorname{ev}_{\tau, \nu_{2}}(x)=0$. On the other hand, the $\mathbb{Z}_{2}$-valued point $x^{\prime}=\left(-\frac{1}{3}: \frac{1}{3}:-\frac{2}{3}: \frac{1}{3}: 1\right) \in \mathscr{U}\left(\mathbb{Z}_{2}\right)$ yields $\left(x_{1}, x_{3}\right)_{2}=\left(\frac{1}{3}, \frac{1}{3}\right)_{2}=-1$, hence $\operatorname{ev}_{\tau, \nu_{2}}(x)=\frac{1}{2}$.

iii) First of all, the integral point $x=(-1: 1: 0:-1: 1) \in U(\mathbb{R})$ yields $\left(x_{1},-x_{3}\right)_{\infty}=(1,1)_{\infty}=1$, therefore $\operatorname{ev}_{\alpha+\tau, \nu_{\infty}}(x)=0$. On the other hand, there is the real point $x^{\prime}=(0:-4: \sqrt{2}: 2: 1)$ such that $\left(x_{1}^{\prime},-x_{3}^{\prime}\right)_{\infty}=(-4,-2)_{\infty}=-1$, hence $\operatorname{ev}_{\alpha+\tau, \nu_{\infty}}(x)=\frac{1}{2}$.

Finally, constancy of $\mathrm{ev}_{\alpha+\tau, \nu_{2}}$ is elementary, too, but quite involved. The idea of the proof is roughly as follows. It suffices to show $\left(x_{1},-x_{3}\right)_{2}=1$ for every $\mathbb{Z}_{2}$-valued point $x=\left(x_{0}: x_{1}: x_{2}: x_{3}: x_{4}\right) \in \mathscr{U}\left(\mathbb{Z}_{2}\right)$ such that $x_{1} \neq 0$ and $x_{3} \neq 0$.

In order to do this, several cases have to be distinguished. It turns out that the assumption $8 \nmid x_{3}$ leads to only finite many cases and that $\left(x_{1},-x_{3}\right)_{2}=1$ is fulfilled in each of them. On the other hand, if $8 \mid x_{3}$ then the second equation $x_{0}^{2}+x_{3}^{2}=\left(1-x_{3}\right) x_{1}$ shows that $\left(x_{1},-1\right)_{2}=1$. Moreover, $x_{1}$ is automatically a square, except for the case that $\left|\nu_{2}\left(x_{0}\right)-\nu_{2}\left(x_{3}\right)\right| \leq 1$. In this case, however, $\nu_{2}\left(x_{0} x_{1}\right) \geq \nu_{2}\left(x_{3}\right)+3$ such that the first equation implies that $x_{3}$ is a square. The assertion follows.

iv) is an immediate consequence of ii) and iii), while v) follows from the fact that $(-1)$ is a square in $\mathbb{Q}_{p}$ for $p \equiv 1(\bmod 4)$.

Remarks 7.3. i) The algebraic Brauer class $\alpha$ causes a violation of strong approximation off $S_{1}:=\{p$ prime $\mid p \equiv 1(\bmod 4)\}$. In fact, a $\mathbb{Z}\left[\frac{1}{S_{1}}\right]$-valued point such that $x_{1} \neq 0$ must necessarily fulfil $\left(\frac{x_{1}}{x_{4}},-1\right)_{2}+\left(\frac{x_{1}}{x_{4}},-1\right)_{\infty}=0$, although not all adelic points outside $S_{1}$ satisfy this relation.

ii) Furthermore, strong approximation off $\{\infty\}$ is violated. In fact, the Brauer class $\tau$ yields a restriction for integral points. Namely, an integral point such that $x_{1} \neq 0$ and $x_{3} \neq 0$ must necessarily fulfil $\left(x_{1}, x_{3}\right)_{2}=1$, although not all $\mathbb{Z}_{2}$-valued points satisfy this relation.

iii) The Brauer class $\alpha+\tau$ yields another restriction for integral points. Indeed, an integral point such that $x_{1} \neq 0$ and $x_{3} \neq 0$ must necessarily fulfil $\left(x_{1},-x_{3}\right)_{\infty}=1$, although there exist real points violating this. 
iv) There are infinitely many integral points on $\mathscr{U}$. In fact, the curves defined by $V\left(X_{3}\right)$ and $V\left(X_{1}+X_{3}\right)$ yield the families

$$
\left(-n^{2}: n^{4}: \pm n^{3}: 0: 1\right) \text { and }\left(-\left(n^{2}+1\right):\left(n^{2}+1\right)^{2}: \pm\left(n^{2}+1\right) n:-\left(n^{2}+1\right)^{2}: 1\right) \text {. }
$$

v) A search for integral points on $\mathscr{U}$ delivered exactly 16 of height $<50000$ that are of neither of the two types above. These are

$(-5: 13: \pm 8:-1: 1),(-58: 676: \pm 198:-4: 1),(-268: 4240: \pm 1064:-4224: 1)$, $(-1297: 11437: \pm 3850:-11289: 1),(-2416: 6736: \pm 4034:-1020: 1),(-4513: 9685: \pm 6611:-3084: 1)$, $(-6668: 13456: \pm 9472:-5824: 1)$, and $(-11681: 27061: \pm 17779:-6700: 1)$.

Example 7.4. Let $\mathscr{X}^{\prime} \subset \mathbf{P}_{\mathbb{Z}}^{4}$ be given by the system of equations

$$
\begin{aligned}
X_{0}\left(4 X_{1}+3 X_{4}\right)+X_{2}^{2} & =X_{4}\left(4 X_{3}+3 X_{4}\right), \\
X_{3}\left(4 X_{1}+4 X_{3}+6 X_{4}\right)+X_{0}^{2} & =X_{4}\left(4 X_{1}+3 X_{4}\right)
\end{aligned}
$$

and put $\mathscr{U}^{\prime}:=\mathscr{X}^{\prime} \backslash \mathscr{H}$, for the hyperplane $\mathscr{H}:=V\left(X_{4}\right) \subset \mathbf{P}_{\mathbb{Z}}^{4}$. Denote the generic fibre of $\mathscr{U}^{\prime}$ by $U^{\prime}$.

Then $\mathscr{U}^{\prime}\left(\mathbb{Z}_{p}\right) \neq \emptyset$ for every prime number $p$ and $U^{\prime}(\mathbb{Q}) \neq \emptyset$, but there are no integral points, $\mathscr{U}^{\prime}(\mathbb{Z})=\emptyset$. In other words, the integral Hasse principle is violated. The violation can be explained by a Brauer-Manin obstruction.

Proof. There is a morphism $\pi: \mathscr{U}^{\prime} \rightarrow \mathscr{U}$ to the scheme from the example before that is given by

$$
\pi:\left(x_{0}: x_{1}: x_{2}: x_{3}: 1\right) \mapsto\left(x_{0}:\left(4 x_{1}+3\right): x_{2}:\left(4 x_{3}+3\right): 1\right)
$$

This morphism yields bijections between the sets of $\mathbb{Q}$-rational points, as well as between the sets of $\mathbb{Z}_{p}$-valued points for $p \neq 2$. In particular, $U^{\prime}(\mathbb{Q}) \neq \emptyset$ and $\mathscr{U}^{\prime}\left(\mathbb{Z}_{p}\right) \neq \emptyset$, except possibly for $p \neq 2$. Moreover, there is a $\mathbb{Z}_{2}$-valued point such that $x_{1}=0$ and $x_{3}=2$.

There is, however, no integral point on $\mathscr{U}^{\prime}$. In fact, the image of such a point would fulfil $\left(4 x_{1}+3,4 x_{3}+3\right)_{2}=(3,3)_{2}=-1$, in contradiction to what was proven, cf. Remark 7.3.i).

Remark 7.5. Again, the $\mathbb{Q}$-scheme $U^{\prime}$ is strongly unobstructed at $\infty$. Concerning this, the situation is completely analogous to the one described in Remark 6.5.i).

Blowing up a point. A cubic surface.

Example 7.6. Let $\mathscr{S} \subset \mathbf{P}_{\mathbb{Z}}^{3}$ be given by the equation

$$
Y_{0}^{3}+Y_{0} Y_{2}^{2}-Y_{1}^{2} Y_{2}+Y_{1}^{2} Y_{3}+Y_{2}^{2} Y_{3}-Y_{2} Y_{3}^{2}=0 .
$$

Put $\mathscr{V}:=\mathscr{S} \backslash \mathscr{E}$ for the hyperplane $\mathscr{E}:=V\left(Y_{3}\right) \subset \mathbf{P}_{\mathbb{Z}}^{3}$.

Then every integral point $\left(y_{0}: y_{1}: y_{2}: 1\right) \in \mathscr{V}(\mathbb{Z})$ such that $y_{0} y_{2} \neq 0$ satisfies that $\left(y_{0}\left(y_{2}-y_{1}^{2}\right), y_{2}\right)_{2}=1$. In particular, strong approximation off $\{\infty\}$ is violated.

Proof. The scheme $\mathscr{S}$ is obtained from the scheme $\mathscr{X}$ from Example 7.1 by blowing up the point $(0: 1: 0: 0: 0)$. We eliminated $X_{1}$ from the equations defining $\mathscr{X}$ and 
replaced the coordinate sections $X_{0}, X_{2}, X_{3}, X_{4}$, in this order, by $Y_{0}, Y_{1}, Y_{2}$, and $Y_{3}$. An integral point on $\mathscr{V}$ corresponds to a $\mathbb{Q}$-rational point $\left(x_{0}: x_{1}: x_{2}: x_{3}: 1\right) \in U(\mathbb{Q})$ such that $x_{0}, x_{2}$, and $x_{3}$ are integers, but $x_{1}$ not necessarily.

For the main assertion, let us first observe that there are no integral points such that $y_{2}-y_{1}^{2}=0$, but $y_{0} y_{2} \neq 0$. Thus, the symbol is properly defined. Moreover, Example 7.2.ii) shows that $\left(y_{0}\left(y_{2}-y_{1}^{2}\right), y_{2}\right)_{\infty}=1$. We will complete the proof by verifying $\left(y_{0}\left(y_{2}-y_{1}^{2}\right), y_{2}\right)_{p}=1$ for every prime $p \neq 2$.

In order to do this, assume that $p \nmid\left(x_{3}-1\right)$ first. Then $\left(x_{3}-1\right) \cdot x_{1}=-\left(x_{0}^{2}+x_{3}^{2}\right)$ implies that $x_{1}$ is a $p$-adic integer. Consequently, by Example 7.2.i), one has $\left(y_{0}\left(y_{2}-y_{1}^{2}\right), y_{2}\right)_{p}=\left(x_{1}, x_{3}\right)_{p}=1$. On the other hand, if $p \mid\left(x_{3}-1\right)$ then $x_{3}$ is a square in $\mathbb{Z}_{p}^{*}$ and therefore $\left(y_{0}\left(y_{2}-y_{1}^{2}\right), y_{2}\right)_{p}=\left(x_{1}, x_{3}\right)_{p}=1$, as well. The assertion follows.

Finally, we note that, for the point $\left(y_{0}: y_{1}: y_{2}: 1\right)=\left(-\frac{1}{3}:-\frac{2}{3}: \frac{1}{3}: 1\right) \in \mathscr{V}\left(\mathbb{Z}_{2}\right)$, one has that $\left(y_{0}\left(y_{2}-y_{1}^{2}\right), y_{2}\right)_{2}=-1$. Hence, this $\mathbb{Z}_{2}$-valued point cannot be approximated by integral ones.

Remarks 7.7. Write $S \subset \mathbf{P}_{\mathbb{Q}}^{3}$ and $E \subset \mathbf{P}_{\mathbb{Q}}^{3}$ for the generic fibres of $\mathscr{S}$ and $\mathscr{E}$, respectively, and put $V:=S \backslash E$. Then, under the blow-down morphism $\pi: S \rightarrow X$, one has $\pi^{-1}(H)=E \cup L$ and, hence, $\pi^{-1}(U)=V \backslash L$, for $L \subset S$ the line given by $Y_{0}=Y_{2}-Y_{3}=0$.

i) The effect shown above is that of the Brauer class $\pi^{*} \tau \in \operatorname{Br}(V \backslash L)$, which, as $\frac{Y_{2}}{Y_{3}}=\frac{X_{3}}{X_{4}}$ is a square on $L$, turns out to be unobstructed at $L$ and extends to the whole of $V$.

ii) The other generator shows a behaviour similar to that indicated in Example 7.6. I.e., it yields that $\operatorname{gcd}\left(y_{0}, y_{2}-1\right)>1$ or $\left(y_{0}\left(y_{2}-y_{1}^{2}\right),-y_{2}\right)_{\infty}=1$ for every integral point, while neither of the two statements is always true.

Example 7.8. Let $\mathscr{S}^{\prime} \subset \mathbf{P}_{\mathbb{Z}}^{3}$ be given by the equation

$$
\begin{aligned}
16 Y_{0}^{3}+12 Y_{0}^{2} Y_{3}+16 Y_{0} Y_{2}^{2}+24 Y_{0} Y_{2} Y_{3}+12 Y_{0} Y_{3}^{2}-4 Y_{1}^{2} Y_{2}-2 Y_{1}^{2} Y_{3}+8 Y_{2}^{2} Y_{3} & \\
+11 Y_{2} Y_{3}^{2}+4 Y_{3}^{3} & =0
\end{aligned}
$$

and put $\mathscr{V}^{\prime}:=\mathscr{S}^{\prime} \backslash \mathscr{E}$, for the hyperplane $\mathscr{E}:=V\left(Y_{3}\right) \subset \mathbf{P}_{\mathbb{Z}}^{3}$.

Then $\mathscr{V}^{\prime}\left(\mathbb{Z}_{p}\right) \neq \emptyset$ for every prime number $p$ and $\mathscr{V}^{\prime}(\mathbb{Q}) \neq \emptyset$, but there are no integral points, $\mathscr{V}^{\prime}(\mathbb{Z})=\emptyset$. In other words, the integral Hasse principle is violated. The violation is explained by a Brauer-Manin obstruction.

Proof. There is a morphism $\pi: \mathscr{V}^{\prime} \rightarrow \mathscr{V}$ to the scheme from Example 7.6 that is given by

$$
\pi:\left(y_{0}: y_{1}: y_{2}: 1\right) \mapsto\left(\left(4 y_{0}+1\right): 2 y_{1}:\left(4 y_{2}+3\right): 1\right)
$$

This morphism yields bijections between the sets of $\mathbb{Q}$-rational points, as well as between the sets of $\mathbb{Z}_{p}$-valued points for $p \neq 2$. In particular, $\mathscr{V}^{\prime}(\mathbb{Q}) \neq \emptyset$ and $\mathscr{V}^{\prime}\left(\mathbb{Z}_{p}\right) \neq \emptyset$, except possibly for $p \neq 2$. Moreover, there is the $\mathbb{Z}_{2}$-valued point $\left(-\frac{1}{3}:-\frac{1}{3}:-\frac{2}{3}: 1\right)$. 
There is, however, no integral point on $\mathscr{V}^{\prime}$. In fact, the image of such a point would fulfil $\left(\left(4 y_{0}+1\right)\left[4 y_{2}+3-4 y_{1}^{2}\right], 4 y_{2}+3\right)_{2}=(3,3)_{2}=-1$, in contradiction to what was proven.

\section{Yet ANOTHER EXAMPLE}

In the example below, algebraic Brauer classes interact with effects caused by $U(\mathbb{R})$ being disconnected into compact and non-compact components. The two non-compact components of $U(\mathbb{R})$ in fact fulfil the requirements of Definition 2.2.ii). Nevertheless, strong approximation off $\{\infty\}$ is violated, as there are Brauer classes $\alpha_{1}$ and $\alpha_{2}$ working at the primes 2,3 , and $\infty$.

Just requiring 2-adic and 3 -adic approximation by a sequence $\left(x_{n}\right)_{n \in \mathbb{N}}$ of integral points, these classes enforce a certain behaviour at the infinite prime, as well. In the present case, they determine the connected component of $U(\mathbb{R})$ the points $x_{n}$ are lying on. The violation of strong approximation off $\{\infty\}$ happens when trying to approximate an adelic point, for which the $x_{n} \in \mathscr{U}(\mathbb{Z})$, for $n \rightarrow \infty$, turn out to be bound to to the single compact component of $U(\mathbb{R})$. This underlines observations made by U. Derenthal and D. Wei in [DW, Example 6.2].

As a consequence, we conclude that every serious definition of unobstructedness at infinity must include requirements on all connected components of $U(\mathbb{R})$. In particular, it is clearly insufficient just to require the existence of a single well-behaved one. Or to require that each irreducible component of the boundary contains limit points just from $U(\mathbb{R})$.

Example 8.1. Let $X \subset \mathbf{P}_{\mathbb{Q}}^{4}$ be given by the system of equations

$$
\begin{aligned}
X_{0}\left(X_{0}+X_{1}\right) & =X_{2}^{2}+\left(X_{0}+X_{4}\right)^{2}, \\
\left(X_{0}+X_{2}\right)\left(X_{0}+2 X_{2}\right) & =2 X_{1}^{2}+3 X_{3}^{2}
\end{aligned}
$$

and put $U:=X \backslash H$, for the hyperplane $H:=V\left(X_{4}\right) \subset \mathbf{P}_{\mathbb{Q}}^{4}$.

i) Then $X$ is a del Pezzo surface of degree four.

ii) The curve $D:=H \cap X$ is non-singular of genus one. One has $D(\mathbb{Q})=\emptyset$, but $D(\mathbb{R}) \neq \emptyset$. Furthermore, $J(D)(\mathbb{Q}) \cong \mathbb{Z}$.

iii) The manifold $X(\mathbb{R})$ consists of two connected components and its submanifold $U(\mathbb{R})$ decomposes into three connected components. $U$ is not unobstructed at $\infty$, not even in the weak sense.

iv) One has $\operatorname{Br}(U) / \operatorname{Br}_{0}(U) \cong(\mathbb{Z} / 2 \mathbb{Z})^{2}$, two generators being represented by

$$
\begin{aligned}
& \alpha_{1}:\left(\frac{X_{0}}{X_{4}},-1 ;-1\right) \quad \text { and } \\
& \alpha_{2}:\left(\frac{X_{0}+X_{2}}{X_{4}},-6 ;-1\right) .
\end{aligned}
$$

Moreover, $\operatorname{Br}(U)=\operatorname{Br}_{1}(U)$. 
Proof. i) and ii) magma reports that both $X$ and $D$ are non-singular. Furthermore, the system of equations defining $D$ is easily seen to be insoluble in $\mathbb{Q}_{3}$. Therefore $D(\mathbb{Q})=\emptyset$. On the other hand, $\left(1: 1: 1: \frac{2}{3} \sqrt{3}: 0\right) \in D(\mathbb{R})$. The Jacobian of $D$ is reported to be isomorphic to the elliptic curve $E: y^{2} z=x^{3}+6 x^{2} z+72 x z^{2}-54 z^{3}$ over $\mathbb{Q}$ of $j$-invariant $\frac{512000}{603}$ and Mordell-Weil group $E(\mathbb{Q})$ being free of rank one.

iii) The pencil of quadrics in $\mathbf{P}^{4}$ defining $X$ contains five degenerate ones, all of which are real. Among the corresponding rank-four discriminants, four are negative and the fifth is positive. According to [VAV, Theorem 3.4 and Lemma 5.1], this implies $\operatorname{Br}\left(X_{\mathbb{R}}\right) / \operatorname{Br}(\mathbb{R})=(\mathbb{Z} / 2 \mathbb{Z})^{2}$, which in itself shows that $X(\mathbb{R})$ consists of exactly two connected components [Si, Théorème 1.4]. The evaluation of the global Brauer class on $X_{\mathbb{R}}$, given by $\left(\frac{X_{0}+X_{2}}{X_{0}},-1 ;-1\right)$, distinguishes the two components.

As the cubic polynomial $x^{3}+6 x^{2}+72 x-54$ has exactly one real root, we see that $D(\mathbb{R})$ is connected. Such a curve may meet only one component of $X(\mathbb{R})$, it obviously meets $X_{+}$, the one where $\frac{X_{0}+X_{2}}{X_{0}}$ is positive, and it cannot cut that into more than two components. On the other hand, one sees that $D(\mathbb{R})$ indeed decomposes $X_{+}$into two components, these being distinguished by the sign of $\frac{X_{0}}{X_{4}}$. Observe that both are non-empty, as they contain the real points $(17: 3: 4:-13: 1)$ and $\left(-\frac{41}{8}: \frac{3}{2}: \frac{5}{4}:-\frac{11}{8}: 1\right)$, respectively.

Finally, let us note once again that the component $X_{-}:=X(\mathbb{R}) \backslash X_{+}$of $X(\mathbb{R})$ is not at all met by the curve $D(\mathbb{R})$. Hence, $X_{-}$forms as well a connected component of $U(\mathbb{R})$. As $X_{-}$is compact, Definition 2.2.i) immediately shows that $U$ cannot be weakly unobstructed.

iv) A calculation using Gröbner bases indicates that the Galois group operating on the 16 lines on $X$ is of order exactly 96. As both quadrics used to describe $X$ are defined over $\mathbb{Q}$ and of rank 4, Theorem 4.8.b.iii) implies that $\operatorname{Br}_{1}(U) / \operatorname{Br}_{0}(U) \cong(\mathbb{Z} / 2 \mathbb{Z})^{2}$.

Moreover, Theorem 5.4 shows that $\left(\frac{X_{0}}{X_{4}},-1 ;-1\right)$ and $\left(\frac{X_{0}+X_{2}}{X_{4}},-6 ;-1\right)$ define nontrivial algebraic Brauer classes on $U$. They do not just differ by an element from $\operatorname{Br}(\mathbb{Q})$, as easily follows from the results given in Example 8.3.i) and ii), below. On the other hand, $J(D)$ do not have any nontrivial $\mathbb{Q}$-rational isogeny. Thus, Corollary 4.10 yields $\operatorname{Br}(U)=\operatorname{Br}_{1}(U)$.

Remarks 8.2. i) Writing down the points on $U$ in the form $\left(x_{0}: x_{1}: x_{2}: x_{3}: 1\right)$, the three connected components of $U(\mathbb{R})$ are distinguished by the signs of $x_{0}$ and $x_{0}+x_{2}$ (respectively that of $x_{0}+2 x_{2}$ if $x_{0}+x_{2}=0$ ).

ii) There are no real points on $U$ such that $x_{0}>0$ and $x_{0}+x_{2}<0$, in accordance with our result that $U(\mathbb{R})$ consists of only three components. One may see this in an elementary fashion, as follows.

The first equation implies $x_{1}=\frac{x_{2}^{2}}{x_{0}}+\frac{1}{x_{0}}+2$, hence $x_{1}>\frac{x_{2}^{2}}{x_{0}}$ in the case that $x_{0}>0$. Moreover, $\left(\frac{x_{0}}{x_{2}}\right)^{2}\left(\frac{x_{0}}{x_{2}}+1\right)\left(\frac{x_{0}}{x_{2}}+2\right)>2$, which implies that $\frac{x_{0}}{x_{2}}<-2.293 \ldots<-1$ if $x_{2}$ is negative, and hence $x_{0}+x_{2}=x_{2}\left(\frac{x_{0}}{x_{2}}+1\right)>0$.

iii) One may as well verify in an elementary manner that the component where $x_{0}<0$ and $x_{0}+x_{2}>0$ is compact. All such real points fulfil $x_{2}<3$. 
Indeed, the equations of $X$ imply that $\left(\frac{x_{0}}{x_{2}}\right)^{2}\left(\frac{x_{0}}{x_{2}}+1\right)\left(\frac{x_{0}}{x_{2}}+2\right) \geq 2\left[1+\frac{1}{x_{2}^{2}}+\frac{2 x_{0}}{x_{2}^{2}}\right]^{2}$. If one assumes $x_{2} \geq 3$ and $\left|x_{0}\right|<\left|x_{2}\right|$ then the right hand side is strictly larger than $2\left[1+0-\frac{2}{3}\right]^{2}=\frac{2}{9}$. However, the polynomial $t^{2}(t+1)(t+2)$ adopts its maximum in the interval $[-1,0]$ at the point $t=\frac{-9+\sqrt{17}}{8}$ and this maximum is $0.201 \ldots<\frac{2}{9}$.

Example 8.3 (continued). Let $X, U, \alpha_{1}$, and $\alpha_{2}$ be as above. Moreover, let $\mathscr{X} \subset \mathbf{P}_{\mathbb{Z}}^{4}$ be defined by the same system of equations as $X$ and put $\mathscr{U}:=\mathscr{X} \backslash \mathscr{H}$, for $\mathscr{H}:=V\left(X_{4}\right) \subset \mathbf{P}_{\mathbb{Z}}^{4}$. Then

i) for all primes $p \neq 2,3, \infty$, the local evaluation maps $\operatorname{ev}_{\alpha_{1}, \nu_{p}}: \mathscr{U}\left(\mathbb{Z}_{p}\right) \rightarrow \mathbb{Q} / \mathbb{Z}$, $\mathrm{ev}_{\alpha_{2}, \nu_{p}}: \mathscr{U}\left(\mathbb{Z}_{p}\right) \rightarrow \mathbb{Q} / \mathbb{Z}$, and $\mathrm{ev}_{\alpha_{1}+\alpha_{2}, \nu_{p}}: \mathscr{U}\left(\mathbb{Z}_{p}\right) \rightarrow \mathbb{Q} / \mathbb{Z}$ are constantly zero.

ii) The local evaluation map $\operatorname{ev}_{\alpha_{1}, \nu_{3}}: \mathscr{U}\left(\mathbb{Z}_{3}\right) \rightarrow \mathbb{Q} / \mathbb{Z}$ is constantly zero, while $\mathrm{ev}_{\alpha_{1}, \nu_{\infty}}: U(\mathbb{R}) \rightarrow \frac{1}{2} \mathbb{Z} / \mathbb{Z}$ and $\mathrm{ev}_{\alpha_{1}, \nu_{2}}: \mathscr{U}\left(\mathbb{Z}_{2}\right) \rightarrow \mathbb{Q} / \mathbb{Z}$ are non-constant.

iii) The local evaluation maps $\operatorname{ev}_{\alpha_{2}, \nu_{\infty}}: U(\mathbb{R}) \rightarrow \frac{1}{2} \mathbb{Z} / \mathbb{Z}, \mathrm{ev}_{\alpha_{2}, \nu_{2}}: \mathscr{U}\left(\mathbb{Z}_{2}\right) \rightarrow \mathbb{Q} / \mathbb{Z}$, and $\mathrm{ev}_{\alpha_{2}, \nu_{3}}: \mathscr{U}\left(\mathbb{Z}_{3}\right) \rightarrow \mathbb{Q} / \mathbb{Z}$ are all non-constant.

iv) The local evaluation maps $\mathrm{ev}_{\alpha_{1}+\alpha_{2}, \nu_{\infty}}: U(\mathbb{R}) \rightarrow \frac{1}{2} \mathbb{Z} / \mathbb{Z}, \mathrm{ev}_{\alpha_{1}+\alpha_{2}, \nu_{2}}: \mathscr{U}\left(\mathbb{Z}_{2}\right) \rightarrow \mathbb{Q} / \mathbb{Z}$, and $\mathrm{ev}_{\alpha_{1}+\alpha_{2}, \nu_{3}}: \mathscr{U}\left(\mathbb{Z}_{3}\right) \rightarrow \mathbb{Q} / \mathbb{Z}$ are all non-constant.

Proof. i) For the $\mathrm{ev}_{\alpha_{1}, \nu_{p}}$, Lemma 5.7 applies, since $d=-1$, the linear forms $X_{0}$, $X_{0}+X_{1}, X_{2}$, and $X_{0}+X_{4}$ are linearly independent modulo an arbitrary prime $p$, and the cusp (0:0:0:1:0) does not satisfy the second equation $X_{0} X_{1}+X_{2}^{2}=X_{4} X_{3}$ modulo any prime $p \neq 3$.

Similarly, for the $\mathrm{ev}_{\alpha_{2}, \nu_{p}}$, one has $d=-6$, the linear forms $2\left(X_{0}+X_{2}\right), X_{0}+2 X_{2}$, $X_{1}$, and $X_{3}$ are linearly independent modulo every prime $p$ and the cusp $(0: 0: 0: 0: 1)$ does not satisfy the first equation $X_{0}\left(X_{0}+X_{1}\right)=X_{2}^{2}+\left(X_{0}+X_{4}\right)^{2}$ modulo any prime. The assertion about $\mathrm{ev}_{\alpha_{1}+\alpha_{2}, \nu_{p}}$ follows.

ii and iii) Constancy of $\mathrm{ev}_{\alpha_{1}, \nu_{3}}$ follows from Lemma 5.7, too, since the cusp $(0: 0: 0: 1: 0)$ is a point not lying on $\mathscr{U}_{3}$.

Furthermore, the asserted non-constancies are directly checked. In order to do this, let us consider the points $x=\left(5: \frac{12}{5}:-1: \frac{2}{5}: 1\right)$ and $x^{\prime}=\left(-\frac{37}{13}: \frac{21}{13}: \frac{4}{13}: \frac{5}{13}: 1\right)$, for which we clearly have that $x, x^{\prime} \in \mathscr{U}\left(\mathbb{Z}_{2}\right) \cap \mathscr{U}\left(\mathbb{Z}_{3}\right) \cap U(\mathbb{R})$. Explicit calculations show that $\mathrm{ev}_{\alpha_{1}, \nu}(x)=\mathrm{ev}_{\alpha_{2}, \nu}(x)=\mathrm{ev}_{\alpha_{1}+\alpha_{2}, \nu}(x)=0$ for any place $\nu$. On the other hand, $\mathrm{ev}_{\alpha_{1}, \nu}\left(x^{\prime}\right)=\frac{1}{2}$ for $\nu=\nu_{\infty}$ and $\nu=\nu_{2}$, as well as $\mathrm{ev}_{\alpha_{2}, \nu}\left(x^{\prime}\right)=\frac{1}{2}$ for $\nu=\nu_{\infty}$, $\nu=\nu_{2}$, and $\nu=\nu_{3}$. This establishes ii) and iii).

iv) As a consequence of ii) and iii), we see that $\mathrm{ev}_{\alpha_{1}+\alpha_{2}, \nu_{3}}$ is non-constant. Moreover, there is the point $x^{\prime \prime}=\left(-\frac{85}{91}:-\frac{15}{91}: \frac{92}{91}: \frac{9}{91}: 1\right) \in \mathscr{U}\left(\mathbb{Z}_{2}\right) \cap U(\mathbb{R})$, for which direct calculations show that $\operatorname{ev}_{\alpha_{1}+\alpha_{2}, \nu}\left(x^{\prime \prime}\right)=\frac{1}{2}$ for $\nu=\nu_{\infty}$ and $\nu=\nu_{2}$. This completes the proof.

Remarks 8.4. i) Similarly to the situation in the examples of the sections above, the Brauer classes $\alpha_{1}$ and $\alpha_{2}$ cause failures of strong approximation.

There is a violation of strong approximation off $S_{1}:=\{p$ prime $\mid p \equiv 1(\bmod 4)\}$, due to $\alpha_{1}$. A $\mathbb{Z}\left[\frac{1}{S_{1}}\right]$-valued point must necessarily fulfil $\left(\frac{x_{0}}{x_{4}},-1\right)_{2}+\left(\frac{x_{0}}{x_{4}},-1\right)_{\infty}=0$, although not all adelic points outside $S_{1}$ satisfy this relation. 
Similarly, $\alpha_{2}$ causes a violation of strong approximation off

$$
S_{2}:=\left\{p \text { prime } \mid\left(\frac{-6}{p}\right)=1\right\}=\{p \text { prime } \mid p \equiv 1,5,7,11(\bmod 24)\} .
$$

A $\mathbb{Z}\left[\frac{1}{S_{2}}\right]$-valued point such that $x_{0}+x_{2} \neq 0$ must necessarily fulfil the nontrivial relation $\left(\frac{x_{0}+x_{2}}{x_{4}},-6\right)_{2}+\left(\frac{x_{0}+x_{2}}{x_{4}},-6\right)_{3}+\left(\frac{x_{0}+x_{2}}{x_{4}},-6\right)_{\infty}=0$.

However, as $U(\mathbb{R})$ is disconnected and one of its connected components is compact, further effects are occurring. Cf. Example 8.5 below.

ii) There is exactly one integral point on $\mathscr{U}$ lying on the compact component of $U(\mathbb{R})$, namely $(-1: 0: 1: 0: 1)$.

A search for integral points on $\mathscr{U}$ delivered the following twelve others.

$(17: 3: 4: \pm 13: 1),(1409: 147:-452: \pm 383: 1),(6305: 12972: 9043: \pm 3550: 1)$, $(17741: 12759: 15044: \pm 20351: 1),(-23293:-2328:-7367: \pm 19622: 1)$, and $(60569: 2052: 11143: \pm 44472: 1)$.

Example 8.5 (2nd continuation). Let $U$ be as above. Then strong approximation off $\{\infty\}$ is violated for $U$.

Proof. Consider the adelic point $\mathbb{x}$ outside $\infty$ that is equal to $(-1: 0: 1: 0: 1)$ at every prime $p \neq 5, \infty$ and equal to $(17: 3: 4: 13: 1)$ at $p=5$. Working with the model $\mathscr{U}$ as above, strong approximation off $\{\infty\}$ would imply that there exists a sequence $\left(x_{n}\right)_{n \in \mathbb{N}}$ of integral points $x_{n} \in \mathscr{U}(\mathbb{Z})$ being convergent to $\mathbb{x}$ simultaneously with respect to the $2-, 3$-, and 5 -adic topologies. The latter ensures that $x_{n} \neq(-1: 0: 1: 0: 1)$ for $n \gg 0$.

Moreover, 2-adic convergence implies that $\operatorname{ev}_{\alpha_{1}, \nu_{2}}\left(x_{n}\right)=\operatorname{ev}_{\alpha_{1}, \nu_{2}}(-1: 0: 1: 0: 1)=\frac{1}{2}$ and $\mathrm{ev}_{\alpha_{2}, \nu_{2}}\left(x_{n}\right)=\mathrm{ev}_{\alpha_{2}, \nu_{2}}(-1: 0: 1: 0: 1)=0$ for $n \gg 0$, while 3-adic convergence enforces $\mathrm{ev}_{\alpha_{2}, \nu_{3}}\left(x_{n}\right)=\mathrm{ev}_{\alpha_{2}, \nu_{3}}(-1: 0: 1: 0: 1)=0$. The relations discussed in Remark 8.4.i) now imply that, necessarily, $\mathrm{ev}_{\alpha_{1}, \nu_{\infty}}\left(x_{n}\right)=\frac{1}{2}$ and $\operatorname{ev}_{\alpha_{2}, \nu_{\infty}}\left(x_{n}\right)=0$.

In other words, $x_{n}$ must be contained in the same connected component of $U(\mathbb{R})$ as the point $(-1: 0: 1: 0: 1)$. This component is, however, compact and does not contain any other integral point.

\section{REFERENCES}

[AM] Artin, M. and Mumford, D.: Some elementary examples of unirational varieties which are not rational, Proc. London Math. Soc. 25 (1972), 75-95

[BCP] Bosma, W., Cannon, J., and Playoust, C.: The Magma algebra system I. The user language, J. Symbolic Comput. 24 (1997), 235-265

[BMT] Brussel, E., McKinnie, K., and Tengan, E.: Indecomposable and noncrossed product division algebras over function fields of smooth $p$-adic curves, Adv. Math. 226 (2011), 4316-4337

[CS] Colliot-Thélène, J.-L. and Skorobogatov, A. N.: Descente galoisienne sur le groupe de Brauer, Journal für die reine und angewandte Mathematik 682 (2013), 141-165

[CW] Colliot-Thélène, J.-L. and Wittenberg, O.: Groupe de Brauer et points entiers de deux familles de surfaces cubiques affines, Amer. J. Math. 134 (2012), 1303-1327

[CX] Colliot-Thélène, J.-L. and Xu, F.: Brauer-Manin obstruction for integral points of homogeneous spaces and representation by integral quadratic forms. With an appendix by D. Wei and Xu, Compos. Math. 145 (2009), 309-363 
[Co] Corn, P.K.: Del Pezzo surfaces and the Brauer-Manin obstruction, Ph.D. thesis, Harvard 2005

[DW] Derenthal, U. and Wei, D.: Strong approximation and descent, Preprint available at http://arxiv.org/abs/1311.3914

[Do] Dolgachev, I. V.: Classical Algebraic Geometry: a modern view, Cambridge University press, Cambridge 2012

[EJ] Elsenhans, A.-S. and Jahnel, J.: On cubic surfaces with a rational line, Archiv der Mathematik 98 (2012), 229-234

[Fi] Finsler, P.: Über das Vorkommen definiter und semidefiniter Formen in Scharen quadratischer Formen, Comment. Math. Helv. 9 (1937), 188-192

[GrBrII] Grothendieck, A.: Le groupe de Brauer II: Théorie cohomologique, Séminaire Bourbaki, $18^{\mathrm{e}}$ année $1965 / 66, \mathrm{n}^{\mathrm{o}} 297$

[GrBrIII] Grothendieck, A.: Le groupe de Brauer III: Exemples et compléments, in: Grothendieck, A.: Dix exposés sur la cohomologie des schémas, North-Holland, Amsterdam and Masson, Paris 1968, 88-188

[Harp] Harpaz, Y.: Geometry and arithmetic of certain log $K 3$ surfaces, Preprint available at http://arxiv.org/abs/1511.01285

[Hart] Hartshorne, R.: Algebraic Geometry, Graduate Texts in Mathematics 52, Springer, New York 1977

[Hu] Humphreys, J.E.: Introduction to Lie algebras and representation theory, Graduate Texts in Mathematics 9, Springer, New York-Berlin 1972

[Ja] Jahnel, J.: Brauer groups, Tamagawa measures, and rational points on algebraic varieties, Mathematical Surveys and Monographs 198, AMS, Providence 2014

[JL] Jahnel. J. and Loughran, D.: The Hasse principle for lines on del Pezzo surfaces, To appear in: International Mathematical Research Notices

[KT] Kresch, A. and Tschinkel, Yu.: Two examples of Brauer-Manin obstruction to integral points, Bull. Lond. Math. Soc. 40 (2008), 995-1001

[KST] Kunyavskij, B. È., Skorobogatov, A.N., and Tsfasman, M. A.: Del Pezzo surfaces of degree four, Mém. Soc. Math. France 37 (1989), 1-113

[Ma] Manin, Yu. I.: Cubic forms, algebra, geometry, arithmetic, North-Holland Publishing Co. and American Elsevier Publishing Co., Amsterdam-London and New York 1974

[Mi] Milne, J. S.: Étale Cohomology, Princeton University Press, Princeton 1980

[No] Nori, M. V.: Zariski's conjecture and related problems, Ann. Sci. École Norm. Sup. 16 (1983), 305-344

[Pi] Pierce, R. S.: Associative algebras, Graduate Texts in Mathematics 88, Springer, New York-Berlin 1982

[PR] Platonov, V. and Rapinchuk, A.: Algebraic groups and number theory, Pure and Applied Mathematics 139, Academic Press, Boston, 1994

[SGA1] Grothendieck, A.: Revêtements étales et groupe fondamental (SGA 1), Lecture Notes Math. 224, Springer, Berlin 1971

[SGA4] Artin, M., Grothendieck, A. et Verdier, J.-L. (avec la collaboration de Deligne, P. et Saint-Donat, B.): Théorie des topos et cohomologie étale des schémas, Séminaire de Géométrie Algébrique du Bois Marie 1963-1964 (SGA 4), Lecture Notes in Math. 269, 270, 305, Springer, Berlin, Heidelberg, New York 1972-1973

[SGA5] Grothendieck, A. (avec la collaboration de Bucur, I., Houzel, C., Illusie, L. et Serre, J.-P.): Cohomologie $l$-adique et Fonctions $L$, Séminaire de Géométrie Algébrique du Bois Marie 1965-1966 (SGA 5), Lecture Notes in Math. 589, Springer, Berlin, Heidelberg, New York 1977

[Sh] Shimada, I.: Remarks on fundamental groups of complements of divisors on algebraic varieties, Kodai Math. J. 17 (1994), 311-319 
[Si] Silhol, R.: Classification birationnelle des surfaces rationnelles réelles, in: Real analytic and algebraic geometry (Trento 1988), Lecture Notes in Math. 1420, Springer, Berlin 1990, 308-324

[Sk99] Skorobogatov, A. N.: Beyond the Manin obstruction, Invent. Math. 135 (1999), 399-424

[Sk01] Skorobogatov, A. N.: Torsors and rational points, Cambridge Tracts in Mathematics 144, Cambridge University Press, Cambridge 2001

[SD] Swinnerton-Dyer, Sir Peter: The Brauer group of cubic surfaces, Math. Proc. Cambridge Philos. Soc. 113 (1993), 449-460

[Ta] Tate, J.: Global class field theory, in: Algebraic number theory, Edited by J. W. S. Cassels and A. Fröhlich, Academic Press and Thompson Book Co., London and Washington $1967,162-203$

[TWMW] Tu, C., Wang, W., Mourrain, B., and Wang, J.: Signature sequence of intersection curve of two quadrics for exact morphological classification, Preprint available at http:// arxiv.org/abs/cs/0701121

[VAV] Várilly-Alvarado, A. and Viray, B.: Arithmetic of del Pezzo surfaces of degree 4 and vertical Brauer groups, Adv. Math. 255 (2014), 153-181

[Wi] Wittenberg, O.: Intersections de deux quadriques et pinceaux de courbes de genre 1, Lecture Notes in Mathematics 1901, Springer, Berlin 2007

[Xu] $\mathrm{Xu}$, F.: Strong approximation for certain quadric fibrations with compact fibers, Adv. Math. 281 (2015), 279-295

Département Mathematik, Univ. Siegen, Walter-Flex-Str. 3, D-57068Siegen, Germany

E-mail address: jahnel@mathematik.uni-siegen.de

$U R L$ : http://www . uni-math.gwdg.de/jahnel

Mathematisch Instituut, Universiteit Utrecht, Budapestlaan 6, NL-3584 CD UtREChT, The NeTherLANDS

E-mail address: d.schindler@uu.nl

$U R L:$ http://www.uu.nl/staff/DSchindler 